
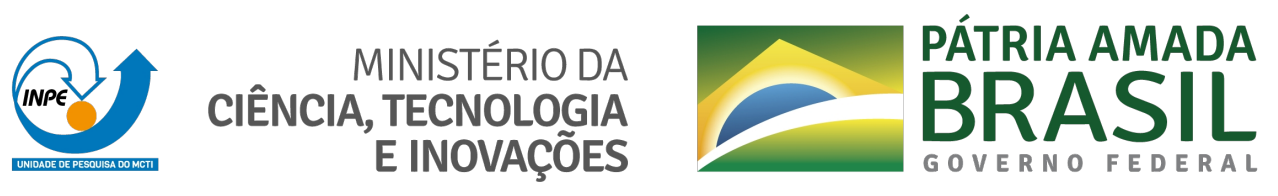

sid.inpe.br/mtc-m21c/2020/12.21.14.31-RPQ

\title{
EXTENDED GRAVITOELECTROMAGNETISM. I. VARIATIONAL FORMULATION
}

Gerson Otto Ludwig

URL do documento original:

<http://urlib.net/8JMKD3MGP3W34R/43QQHDH>

INPE

São José dos Campos

2020 


\section{PUBLICADO POR:}

Instituto Nacional de Pesquisas Espaciais - INPE

Coordenação de Ensino, Pesquisa e Extensão (COEPE)

Divisão de Biblioteca (DIBIB)

CEP 12.227-010

São José dos Campos - SP - Brasil

Tel.:(012) 3208-6923/7348

E-mail: pubtc@inpe.br

\section{CONSELHO DE EDITORAÇÃO E PRESERVAÇÃO DA PRODUÇÃO INTELECTUAL DO INPE - CEPPII (PORTARIA No 176/2018/SEI- INPE):}

\section{Presidente:}

Dra. Marley Cavalcante de Lima Moscati - Divisão de Modelagem Numérica do Sistema Terrestre (DIMNT)

\section{Membros:}

Dra. Carina Barros Mello - Coordenação de Pesquisa Aplicada e Desenvolvimento Tecnológico (COPDT)

Dr. Alisson Dal Lago - Divisão de Heliofísica, Ciências Planetárias e Aeronomia (DIHPA)

Dr. Evandro Albiach Branco - Divisão de Impactos, Adaptação e Vulnerabilidades (DIIAV)

Dr. Evandro Marconi Rocco - Divisão de Mecânica Espacial e Controle (DIMEC)

Dr. Hermann Johann Heinrich Kux - Divisão de Observação da Terra e Geoinformática (DIOTG)

Dra. Ieda Del Arco Sanches - Divisão de Pós-Graduação - (DIPGR)

Silvia Castro Marcelino - Divisão de Biblioteca (DIBIB)

\section{BIBLIOTECA DIGITAL:}

Dr. Gerald Jean Francis Banon

Clayton Martins Pereira - Divisão de Biblioteca (DIBIB)

\section{REVISÃO E NORMALIZAÇÃO DOCUMENTÁRIA:}

Simone Angélica Del Ducca Barbedo - Divisão de Biblioteca (DIBIB)

André Luis Dias Fernandes - Divisão de Biblioteca (DIBIB)

\section{EDITORAÇÃO ELETRÔNICA:}

Ivone Martins - Divisão de Biblioteca (DIBIB)

Cauê Silva Fróes - Divisão de Biblioteca (DIBIB) 

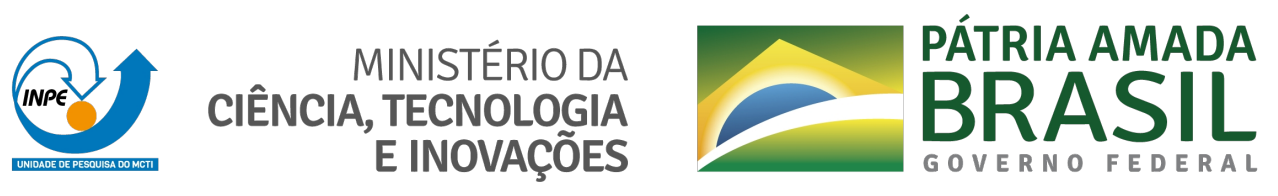

sid.inpe.br/mtc-m21c/2020/12.21.14.31-RPQ

\section{EXTENDED GRAVITOELECTROMAGNETISM. I. VARIATIONAL FORMULATION}

Gerson Otto Ludwig

URL do documento original:

<http://urlib.net/8JMKD3MGP3W34R/43QQHDH>

INPE

São José dos Campos

2020 


\section{(C) $\underset{8}{(1)(9)}$}

Esta obra foi licenciada sob uma Licença Creative Commons Atribuição-NãoComercial 3.0 Não Adaptada.

This work is licensed under a Creative Commons Attribution-NonCommercial 3.0 Unported License. 


\title{
Extended gravitoelectromagnetism. I. Variational formulation
}

\author{
G.O. Ludwig \\ National Institute for Space Research, 12227-010 São José dos Campos, SP, Brazil, \\ National Commission for Nuclear Energy, 22294-900 Rio de Janeiro, RJ, Brazil
}

(Dated: March, 2018 - June, 2020)

\begin{abstract}
Gravitoelectromagnetic (GEM) field theory is considered in the framework of Hamilton's principle. A variational formulation based on this principle describes the dynamics of a fully-relativistic perfect fluid in the presence of the gravitoelectromagnetic field in flat space, leading to the definition of the fluid and field energy-momentum tensors. A fully relativistic Cauchy invariant for a compressible fluid immersed in the gravitoelectromagnetic field is demonstrated. The gravitoelectromagnetic fluid equations of motion are written in covariant form suited for calculating higher-order relativistic effects. The integral form of the conservation theorems is presented, as well as the equations that describe the excitation of gravitoelectromagnetic waves.
\end{abstract}

\section{INTRODUCTION}

The history of gravitoelectromagnetism can be divided into the pre- and post-general relativity periods. The pregeneral relativity period is marked by the prediction of the existence of the planet Neptune by Urbain Le Verrier in 1846 [1]. Le Verrier predicted with a precision of $1^{\circ}$ the position of the then unknow Neptune comparing discrepancies between the observed orbit of the planet Uranus and his calculations of the perturbations introduced by Jupiter and Saturn on Uranus's orbit. This achievement marked the apex of Newton's theory and validated the field of celestial mechanics. Nevertheless, by including the perturbations introduced by the outer planets in the orbit of Mercury, Le Verrier found in 1859 a definitive discrepancy of 38" / century compared with the observed values of the precession of Mercury's perihelion [1] (this value was later corrected for small errors in the planetary masses to 43" / century). This discrepancy remained inexplicable within the Newtonian framework.

Meanwhile, during the second half of the 19th century electromagnetic theory was put on firm footing. The formal analogy between gravitation and electromagnetism led to the development of gravitoelectromagnetism (GEM). First, the notion of a gravitoelectric (GE) field arose naturally from the analogy between the Newtonian and Coulomb potentials, motivating Maxwell to conjecture about the nature of gravitational interactions [2]. Next, it was also natural to attribute a current and a gravitomagnetic (GM) component to moving masses. This notion was used by Holzmüller [3] and Tisserand [4] to analyze the motion of a test mass around a central body based on Newton's attractive potential corrected for a velocity dependent term. The velocity term was introduced in the force of attraction according to Weber electrodynamics, resulting in an elliptical precessing orbit. With this theory Tisserand obtained a precession rate correction for Mercury's perihelion of about 14" / century. This interesting result is possible worth a new examination, in the light of relativity, of the correction introduced by Weber's theory in the Newtonian potential. Undoubtedly, the most elegant analogy between gravitational and electromagnetic theories was made by Heaviside [5]. This analogy included the possibility of gravitational waves propagating, presumably, at the speed of light.

The year of 1916 marked the post-general relativity period when Einstein explained Mercury's perihelion shift of 43" per century, corresponding exactly to astronomical observations [6-8]. The first computation of gravitomagnetic fields produced by rotating masses, according to general relativity, was made by Thirring and Lense [9-11]. The gravitoelectromagnetic equations, which correspond to a weak field approximation to the general relativity field equations, were henceforth developed by Thirring $[12,13]$. This linear approach to gravitoelectromagnetism was reviewed by Mashhoon [14] and is extensively adopted in the literature $[15,16]$. However, the traditional ansatz for the metric perturbations, neglecting higher order terms in the speed of light and imposing four gauge-conditions to the metric tensor in vacuum, leads to analogies between gravity and electromagnetism that hold only for quasi-static fields, providing no support for gravitational waves. Moreover, the equation of motion for a test particle, derived according to the traditional gravitomagnetic theory, does not explain the relativistic correction in the perihelion of Mercury, although this limitation was pointed out in the original work [10]. Some of the weak points of the traditional approach have been partially removed by using alternative ansatzes and additional tensorial terms in the metric perturbation [17], but after one century much of the difficulties remain.

Taking a different approach, the present three-parts work is based on an hydrodynamic variational formulation of the gravitoelectromagnetic equations and the space-time curvature effects in the context of gravitoelectromagnetism. First, the variational approach leads to a consistent set of dynamic equations describing the interaction between matter and the gravitoelectromagnetic field in flat space. This result, which is the main topic of the first part of the present three-parts work, is usually sufficient for describing weak relativistic gravitational phenomena, such as the observed shape of the galactic rotation curve [18]. The metric tensor perturbations introduced by the field-matter interaction, 
both inside the fluid and in the vacuum region, form the subject of the second part of this series. These metric-tensor perturbations lead to a geodesic equation which correctly gives the relativistic correction of the perihelion precession rate of planetary orbits. The application of the geodesic equation to the relativistic correction of Mercury's perihelion precession is the subject of the third part article of the entire work.

Following the program outlined in the preceding paragraph, the equations of motion describing the interaction of a fully-relativistic perfect fluid with the gravitoelectromagnetic field in flat space are derived in Section II. This derivation uses a variational procedure based on Hamilton's, or stationary action, principle. The variations are carried out within the Eulerian framework in Subsection II A. The variation in Lagrangian coordinates is carried out in Subsection II B, leading to the Hamiltonian density and related energy integral in Subsection II C. A hydrogravitomagnetic Cauchy invariant is demonstrated in Section III. In Section IV, the gravitoelectromagnetic fluid equations are written in covariant form. The appropriate forms of the fluid and field energy-momentum tensors are also introduced, as well as the Lorentz transformation properties of the gravitoelectromagnetic field. The conservation equations are presented in Section V, both in differential and integral form, emphasizing a general form of the virial theorem. The excitation of gravitoelectromagnetic waves is discussed in Section VI. Section VII addresses the topics of boundary conditions at the fluid-vacuum interface and of energy density balance inside the fluid. Finally, Section VIII gives the conclusions. The development in the present article repeats most of the work performed in a variational formulation of plasma dynamics [19], due to the analogy between the two problems. Nevertheless, it forms the basis for the second part article and a complete reference for further work.

\section{HAMILTON'S PRINCIPLE AND GRAVITOELECTROMAGNETIC FLUID EQUATIONS}

Hamilton's principle provides an elegant derivation of the equations of motion of a fluid [20-25]. The variational principle in the Eulerian form is presented in Subsection II A. The variational principle in Lagrangian form is discussed in Subsection IIB, leading to the definition of the Hamiltonian density and its related energy integral in Subsection II C. The basic formalism of Hamilton's principle applied to fluid motion is briefly presented next.

In the Eulerian form (fixed frame) Hamilton's principle states that the fluid equations of motion for generalized field coordinates $\varphi(\boldsymbol{r}, t)$ can be derived from a Lagrangian density $\mathcal{L}(\varphi, \dot{\varphi}, \boldsymbol{\nabla} \varphi, t)$ by the variational principle $[26]$

$$
\delta \int d^{3} r d t \mathcal{L}=0
$$

The virtual displacements are constructed for constant $\boldsymbol{r}$ and $t$ so that Hamilton's principle and integration by parts give

$$
\int d^{3} r d t\left[\frac{\partial \mathcal{L}}{\partial \varphi}-\frac{\partial}{\partial t}\left(\frac{\partial \mathcal{L}}{\partial \dot{\varphi}}\right)-\nabla \cdot\left(\frac{\partial \mathcal{L}}{\partial(\boldsymbol{\nabla} \varphi)}\right)\right] \delta \varphi=0
$$

For arbitrary variation of the field coordinate $\varphi$, with vanishing variation $\delta \varphi$ at the end points, this leads to the Euler-Lagrange equation

$$
\frac{\partial \mathcal{L}}{\partial \varphi}=\frac{\partial}{\partial t}\left(\frac{\partial \mathcal{L}}{\partial \dot{\varphi}}\right)+\nabla \cdot\left(\frac{\partial \mathcal{L}}{\partial(\boldsymbol{\nabla} \varphi)}\right)
$$

\section{A. Eulerian variational principle}

The Lagrangian density for a relativistic perfect fluid in the gravitoelectromagnetic approximation is taken to be

$$
\mathcal{L}=-\frac{n m c^{2}}{\gamma}-\frac{p}{\gamma_{A}-1}-\rho \phi_{g}+\boldsymbol{j} \cdot \boldsymbol{A}_{g}-\frac{E_{g}^{2}-c^{2} B_{g}^{2}}{8 \pi G},
$$

or, in covariant form,

$$
\mathcal{L}=-\stackrel{\circ}{U}+j_{\mu} A_{g}^{\mu}+\frac{c^{2}}{16 \pi G} F_{\mu \nu} F^{\mu \nu} .
$$

The various terms of the Lagrangian density are defined in the following. 
The proper energy density $\stackrel{\circ}{U}$, sum of the rest mass and thermal energy densities, is given by

$$
\stackrel{\circ}{U}=-\stackrel{\circ}{n} m u_{\mu} u^{\mu}+\frac{p}{\gamma_{A}-1},
$$

where $u^{\mu}=\gamma(c, \boldsymbol{u})$ is the fluid four-velocity with $u_{\mu}=\eta_{\mu \nu} u^{\nu}=\gamma(-c, \boldsymbol{u})$ and $u_{\mu} u^{\mu}=-c^{2}$. The upper circle label denotes the rest frame. The Minkowski metric tensor is

$$
\eta_{\mu \nu}=\left(\begin{array}{cc}
-1 & \mathbf{0} \\
\mathbf{0} & \overline{\bar{I}}
\end{array}\right)=\eta^{\mu \nu}
$$

and the Lorentz factor is $\gamma=1 / \sqrt{1-\beta^{2}}=1 / \sqrt{1-u^{2} / c^{2}}$, so that the number density transforms from the rest frame to the frame moving with the fluid velocity $\boldsymbol{u}$ as $n=\gamma \stackrel{\circ}{n}$. The absolute temperature transforms as $T=\stackrel{\circ}{T} / \gamma$, the invariant fluid pressure is given by $p=\stackrel{\circ}{n} k_{B} \stackrel{\circ}{T}=n k_{B} T$, and the "adiabatic" coefficient $\gamma_{A}$ is a function of the fluid temperature [19]. The double over-bar denotes a dyadic.

The term $j_{\mu} A_{g}^{\mu}$ corresponds to the interaction energy between the fluid and the gravitoelectromagnetic field

$$
j_{\mu} A_{g}^{\mu}=(-\rho c, \boldsymbol{j}) \cdot\left(\frac{\phi_{g}}{c}, \boldsymbol{A}_{g}\right)=-\rho \phi_{g}+\boldsymbol{j} \cdot \boldsymbol{A}_{g}
$$

where $\rho=n m=\gamma \stackrel{\circ}{n} m$ is the mass density of the fluid, $\boldsymbol{j}=\rho \boldsymbol{u}$ is the mass current density, and $A_{g}^{\mu}=\left(\phi_{g} / c, \boldsymbol{A}_{g}\right)$ is the gravitoelectromagnetic four-vector potential. The gravitoelectromagnetic field variables $\boldsymbol{E}_{g}$ and $\boldsymbol{B}_{g}$ are related to the potentials $\phi_{g}$ and $\boldsymbol{A}_{g}$ by

$$
\begin{aligned}
& \boldsymbol{E}_{g}=-\boldsymbol{\nabla} \phi_{g}-\frac{\partial \boldsymbol{A}_{g}}{\partial t}, \\
& \boldsymbol{B}_{g}=\boldsymbol{\nabla} \times \boldsymbol{A}_{g} .
\end{aligned}
$$

These relations lead to the gravitoelectromagnetic Faraday's law and to the gravitomagnetic Gauss's law, respectively,

$$
\begin{aligned}
\boldsymbol{\nabla} \times \boldsymbol{E}_{g} & =-\frac{\partial \boldsymbol{B}_{g}}{\partial t} \\
\boldsymbol{\nabla} \cdot \boldsymbol{B}_{g} & =0
\end{aligned}
$$

Note that the potential $\boldsymbol{A}_{g}$ has been introduced without the factor $1 / 2$ frequently adopted in gravitoelectromagnetism. The present definition simplifies the comparison with electromagnetic theory.

The free-field Lagrangian is given in terms of the field strengths through the gravitoelectromagnetic field tensor given by

$$
F^{\mu \nu}=\left(\begin{array}{cc}
0 & \boldsymbol{E}_{g} / c \\
-\boldsymbol{E}_{g} / c & \overline{\overline{\boldsymbol{\epsilon}}} \cdot \boldsymbol{B}_{g}
\end{array}\right)
$$

and

$$
F_{\mu \nu}=\eta_{\mu \rho} F^{\rho \sigma} \eta_{\sigma \nu}=\left(\begin{array}{cc}
0 & -\boldsymbol{E}_{g} / c \\
\boldsymbol{E}_{g} / c & \overline{\overline{\boldsymbol{\epsilon}}} \cdot \boldsymbol{B}_{g}
\end{array}\right)
$$

where $\overline{\overline{\boldsymbol{\epsilon}}}$ is the totally antisymmetric Levi-Civita tensor in three dimensions. Noting that (the superscript $T$ denotes the transposed dyadic)

$$
\begin{aligned}
& \left(\overline{\overline{\boldsymbol{\epsilon}}} \cdot \boldsymbol{B}_{g}\right) \cdot\left(\overline{\overline{\boldsymbol{\epsilon}}} \cdot \boldsymbol{B}_{g}\right)^{T}=B_{g}^{2} \overline{\overline{\boldsymbol{I}}}-\boldsymbol{B}_{g} \boldsymbol{B}_{g}, \\
& \left(\overline{\overline{\boldsymbol{\epsilon}}} \cdot \boldsymbol{B}_{g}\right):\left(\overline{\overline{\boldsymbol{\epsilon}}} \cdot \boldsymbol{B}_{g}\right)^{T}=2 B_{g}^{2}
\end{aligned}
$$

the first gravitoelectromagnetic field invariant is

$$
\Lambda_{g}=F_{\mu \nu} F^{\mu \nu}=\left(\begin{array}{cc}
0 & -\boldsymbol{E}_{g} / c \\
\boldsymbol{E}_{g} / c & \overline{\overline{\boldsymbol{\epsilon}}} \cdot \boldsymbol{B}_{g}
\end{array}\right):\left(\begin{array}{cc}
0 & \boldsymbol{E}_{g} / c \\
-\boldsymbol{E}_{g} / c & \overline{\overline{\boldsymbol{\epsilon}}} \cdot \boldsymbol{B}_{g}
\end{array}\right)^{T}=-\frac{2\left(E_{g}^{2}-c^{2} B_{g}^{2}\right)}{c^{2}} .
$$


The pseudo tensor dual of $F^{\mu \nu}$ is

$$
F^{* \mu \nu}=\frac{1}{2} \epsilon^{\mu \nu \rho \sigma} F_{\rho \sigma}=\left(\begin{array}{cc}
0 & \boldsymbol{B}_{g} \\
-\boldsymbol{B}_{g} & -\overline{\overline{\boldsymbol{\epsilon}}} \cdot \boldsymbol{E}_{g} / c
\end{array}\right),
$$

so that

$$
F^{\mu \nu}=-\frac{1}{2} \epsilon^{\mu \nu \rho \sigma} F_{\rho \sigma}^{*}
$$

Using the relation

$$
\left(\overline{\overline{\boldsymbol{\epsilon}}} \cdot \boldsymbol{B}_{g}\right):\left(\overline{\overline{\boldsymbol{\epsilon}}} \cdot \boldsymbol{E}_{g}\right)^{T}=2 \boldsymbol{E}_{g} \cdot \boldsymbol{B}_{g}
$$

the second gravitoelectromagnetic field invariant becomes

$$
M_{g}=F^{\mu \nu} F_{\mu \nu}^{*}=-\frac{4\left(\boldsymbol{E}_{g} \cdot \boldsymbol{B}_{g}\right)}{c} \Longrightarrow \operatorname{det}\left(F^{\mu \nu}\right)=\frac{\left(\boldsymbol{E}_{g} \cdot \boldsymbol{B}_{g}\right)^{2}}{c^{2}}=\left(\frac{M_{g}}{4}\right)^{2} \text {. }
$$

The free-field Lagrangian can be written in terms of the first gravitoelectromagnetic field invariant $\Lambda_{g}$ as

$$
\frac{c^{2}}{16 \pi G} F_{\mu \nu} F^{\mu \nu}=-\frac{E_{g}^{2}-c^{2} B_{g}^{2}}{8 \pi G}=\frac{c^{2} \Lambda_{g}}{16 \pi G}
$$

The introduction of terms depending on $M_{g}$ in the Lagrangian would lead to anisotropy in the fluid-field equations of motion, but this will be disregarded in the present work.

Note that the Lagrangian density $\mathcal{L}$ includes, besides the proper energy of the fluid, the interaction energy between the fluid and the gravitoelectromagnetic field, and the terms related to the free-field energy. This formulation includes all the terms that describe the dynamics of a fluid in the context of the special theory of relativity, i.e., in flat-space. In this form the variational procedure reinstates the equivalence of mass and energy.

The gravitoelectromagnetic equations of motion must be obtained from the expression of the Lagrangian density. The field coordinates are:

$$
\begin{aligned}
\phi_{g}, \boldsymbol{A}_{g} & - \text { gravitoelectromagnetic field potentials } \\
\boldsymbol{u} & - \text { fluid velocity } \\
n & - \text { fluid number density } \\
s & - \text { proper entropy of a fluid element } \\
\boldsymbol{r}_{0} & - \text { Lagrangian coordinate of a fluid element }
\end{aligned}
$$

The vector field $\boldsymbol{r}_{0}(\boldsymbol{r}, t)$ establishes the initial position of the fluid element that occupies the position $\boldsymbol{r}$ at time $t$. The above field variables are not completely independent; they must satisfy the following constraints:

$$
\begin{aligned}
\dot{n}+\nabla \cdot(n \boldsymbol{u}) & =0-\text { continuity condition } \\
d s / d t & =0-\text { condition for isentropic flow } \\
d \boldsymbol{r}_{0} / d t & =0-\text { conservation of the identity of particles }
\end{aligned}
$$

Here $\dot{n}=\partial n / \partial t$ denotes the partial time derivative of $n$. It gives the rate of change of the density $n$ at a stationary point $\boldsymbol{r}$. The total or convective time derivative $d / d t \equiv \partial / \partial t+\boldsymbol{u} \cdot \boldsymbol{\nabla}$, gives the rate of change of a quantity moving instantaneously with the velocity $\boldsymbol{u}$. It describes the advection by fluid motion. As for the fluid constraints [19]: (1) Fluid continuity is a kinematic condition of fluid motion which corresponds to conservation of the number of particles; (2) The specific entropy $s$ of a perfect fluid is constant in time at any point that moves along with the fluid without irreversible processes taking place. This condition is equivalent to energy conservation; (3) The last condition above implies that there is a one-to-one correspondence between Lagrangian and Eulerian coordinates. This constraint was introduced by C.-C. Lin so that the total set of constraints gives rise to a Herivel-Lin flow [21]. Hence, Hamilton's principle can be put in the form

$$
\delta \int d^{3} r d t \underbrace{\left[\mathcal{L}+a m[\dot{n}+\nabla \cdot(n \boldsymbol{u})]+b n m \frac{d s}{d t}+n m \mathbf{c} \cdot\left(\frac{d \boldsymbol{r}_{0}}{d t}\right)\right]}_{\mathcal{L}^{\prime}}=0,
$$


where $a, b$ and $\mathbf{c}$ constitute a set of Lagrangian multipliers.

Now, $\phi_{g}, \boldsymbol{A}_{g}, \boldsymbol{u}, n, s$ and $\boldsymbol{r}_{0}$ are independent functions of $\boldsymbol{r}$ and $t$ that can be varied. In carrying out the variation the volume of integration is kept fixed, in the Eulerian sense. Furthermore, in the rest frame the thermodynamic potentials satisfy the second law

$$
\stackrel{\circ}{T} d s=d(\stackrel{\circ}{U} / \stackrel{\circ}{n})+p d(1 / \stackrel{\circ}{n}) .
$$

In a moving frame this relation becomes

$$
\gamma T d s=d\left(\frac{p}{\gamma_{A}-1} \frac{\gamma}{n}\right)+p d\left(\frac{\gamma}{n}\right) .
$$

Taking into account this fundamental thermodynamic relation, only one equation of state is needed to close the system of fluid equations. For a perfect fluid the equation of state is the ideal gas law $p=n k_{B} T$, so that

$$
\frac{1}{k_{B}} d s=\frac{1}{k_{B} T} d\left(\frac{k_{B} T}{\gamma_{A}-1}\right)+\frac{\gamma_{A}}{\gamma_{A}-1} \frac{d \gamma}{\gamma}-\frac{d n}{n} .
$$

The derivation of the equations of motion from the constrained Lagrangian

$$
\begin{aligned}
\mathcal{L}^{\prime}= & -\frac{n m c^{2}}{\gamma}-\frac{n k_{B} T}{\gamma_{A}-1}-n m \phi_{g}+n m \boldsymbol{u} \cdot \boldsymbol{A}_{g}-\frac{E_{g}^{2}-c^{2} B_{g}^{2}}{8 \pi G} \\
& +a m(\dot{n}+\boldsymbol{u} \cdot \boldsymbol{\nabla} n+n \boldsymbol{\nabla} \cdot \boldsymbol{u})+b n m(\dot{s}+\boldsymbol{u} \cdot \boldsymbol{\nabla} s) \\
& +n m \mathbf{c} \cdot\left(\dot{\boldsymbol{r}_{0}}+\boldsymbol{u} \cdot \boldsymbol{\nabla} \boldsymbol{r}_{0}\right),
\end{aligned}
$$

can be carried out writing an Euler-Lagrange equation for each field coordinate $\varphi=\left(\phi_{g}, \boldsymbol{A}_{g}, \boldsymbol{u}, n, s, \boldsymbol{r}_{0}\right)$ as follows:

$$
\text { (i) } \delta \phi_{g}\left\{\begin{aligned}
\frac{\partial \mathcal{L}^{\prime}}{\partial \phi_{g}} & =-n m=-\rho \\
\frac{\partial \mathcal{L}^{\prime}}{\partial \dot{\phi}_{g}} & =0 \\
\frac{\partial \mathcal{L}^{\prime}}{\partial\left(\boldsymbol{\nabla} \phi_{g}\right)} & =\frac{\partial \mathcal{L}^{\prime}}{\partial \boldsymbol{E}_{g}} \cdot \frac{\partial \boldsymbol{E}_{g}}{\partial\left(\boldsymbol{\nabla} \phi_{g}\right)}=\frac{\partial \mathcal{L}^{\prime}}{\partial \boldsymbol{E}_{g}} \cdot(-\overline{\bar{I}})=-\frac{\partial \mathcal{L}^{\prime}}{\partial \boldsymbol{E}_{g}}=\frac{\boldsymbol{E}_{g}}{4 \pi G}
\end{aligned}\right.
$$

The Euler-Lagrange equation for $\phi_{g}$ gives the GE Gauss' law

$$
\begin{aligned}
& \boldsymbol{\nabla} \cdot \boldsymbol{E}_{g}=-4 \pi G \rho . \\
& (i \boldsymbol{i}) \delta \boldsymbol{A}_{g}\left\{\begin{aligned}
\frac{\partial \mathcal{L}^{\prime}}{\partial \boldsymbol{A}_{g}} & =n m \boldsymbol{u}=\boldsymbol{j} \\
\frac{\partial \mathcal{L}^{\prime}}{\partial \dot{\boldsymbol{A}_{g}}} & =\frac{\partial \mathcal{L}^{\prime}}{\partial \boldsymbol{E}_{g}} \cdot \frac{\partial \boldsymbol{E}_{g}}{\partial \dot{\boldsymbol{A}_{g}}}=-\frac{\boldsymbol{E}_{g}}{4 \pi G} \cdot(-\overline{\overline{\boldsymbol{I}}})=\frac{\boldsymbol{E}_{g}}{4 \pi G} \\
\frac{\partial \mathcal{L}^{\prime}}{\partial\left(\boldsymbol{\nabla} \boldsymbol{A}_{g}\right)} & =\frac{\partial \mathcal{L}^{\prime}}{\partial \boldsymbol{B}_{g}} \cdot \frac{\partial \boldsymbol{B}_{g}}{\partial\left(\boldsymbol{\nabla} \boldsymbol{A}_{g}\right)}=\frac{c^{2} \boldsymbol{B}_{g}}{4 \pi G} \cdot \frac{\partial\left(\boldsymbol{\nabla} \times \boldsymbol{A}_{g}\right)}{\partial\left(\boldsymbol{\nabla} \boldsymbol{A}_{g}\right)} \\
& =\frac{c^{2} \boldsymbol{B}_{g}}{4 \pi G} \cdot\left(-\frac{\overline{\overline{\boldsymbol{\epsilon}}}: \partial\left(\boldsymbol{\nabla} \boldsymbol{A}_{g}\right)}{\partial\left(\boldsymbol{\nabla} \boldsymbol{A}_{g}\right)}\right)=-\frac{c^{2} \boldsymbol{B}_{g}}{4 \pi G} \cdot \overline{\overline{\boldsymbol{\epsilon}}}
\end{aligned}\right.
\end{aligned}
$$

Noting that

$$
\boldsymbol{\nabla} \cdot\left(\boldsymbol{B}_{g} \cdot \overline{\overline{\boldsymbol{\epsilon}}}\right)=\boldsymbol{\nabla} \times \boldsymbol{B}_{g},
$$

the Euler-Lagrange equation for $\boldsymbol{A}_{g}$ gives the GEM Ampère's law

$$
\boldsymbol{\nabla} \times \boldsymbol{B}_{g}=-\frac{4 \pi G}{c^{2}} \boldsymbol{j}+\frac{1}{c^{2}} \frac{\partial \boldsymbol{E}_{g}}{\partial t} .
$$




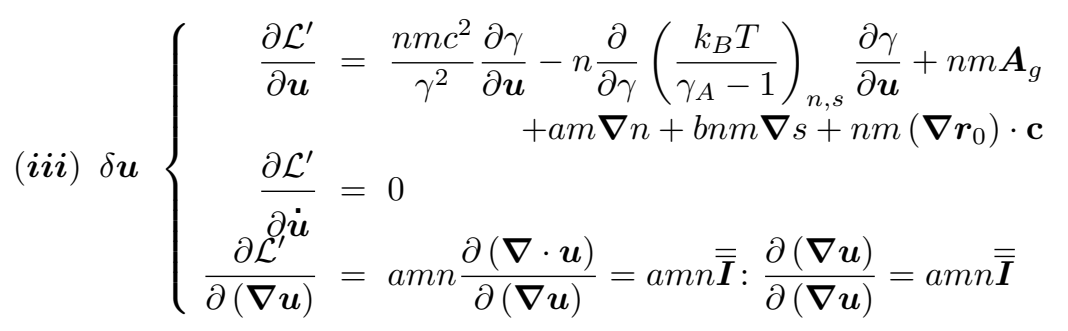

Using

$$
\frac{\partial \gamma}{\partial \boldsymbol{u}}=\gamma^{3} \frac{\boldsymbol{u}}{c^{2}}, \boldsymbol{\nabla} \cdot(a m n \overline{\overline{\boldsymbol{I}}})=m n \boldsymbol{\nabla} a+m a \boldsymbol{\nabla} n
$$

the Euler-Lagrange equation for $\boldsymbol{u}$ gives the fluid velocity in terms of the vector potential $\boldsymbol{A}_{g}$, the scalar potentials $a$ and $s$, and the deformations (strains and rotations) in the initial fluid positions $\boldsymbol{r}_{0}$

$$
\gamma \boldsymbol{u}-\gamma^{3} \frac{\partial}{\partial \gamma}\left(\frac{k_{B} T}{\gamma_{A}-1}\right)_{n, s} \frac{\boldsymbol{u}}{m c^{2}}+\boldsymbol{A}_{g}-\nabla a+b \boldsymbol{\nabla} s+\left(\boldsymbol{\nabla} \boldsymbol{r}_{0}\right) \cdot \mathbf{c}=0
$$

Defining a relativistic factor $\alpha$ such that

$$
\alpha \boldsymbol{u}=\gamma\left[1-\frac{\gamma^{2}}{m c^{2}} \frac{\partial}{\partial \gamma}\left(\frac{k_{B} T}{\gamma_{A}-1}\right)_{n, s}\right] \boldsymbol{u},
$$

the Euler-Lagrange equation $(\boldsymbol{i i \boldsymbol { i }})$ can be written in the compact form

$$
\alpha \boldsymbol{u}+\boldsymbol{A}_{g}=\nabla a-b \nabla s-\mathbf{c} \cdot\left(\boldsymbol{\nabla} \boldsymbol{r}_{0}\right)^{T},
$$

which has the form of a Clebsch representation for the canonical momentum $\boldsymbol{P}=m\left(\alpha \boldsymbol{u}+\boldsymbol{A}_{g}\right)$ of the fluid element.

$$
(\boldsymbol{i v}) \delta n\left\{\begin{aligned}
\frac{\partial \mathcal{L}^{\prime}}{\partial n}= & -\frac{m c^{2}}{\gamma}-\frac{k_{B} T}{\gamma_{A}-1}-n \frac{\partial}{\partial n}\left(\frac{k_{B} T}{\gamma_{A}-1}\right)_{\gamma, s}-m \phi_{g}+m \boldsymbol{u} \cdot \boldsymbol{A}_{g} \\
& +a m \boldsymbol{\nabla} \cdot \boldsymbol{u}+b m \frac{d s}{d t}+m \mathbf{c} \cdot\left(\frac{d \boldsymbol{r}_{0}}{d t}\right) \\
\frac{\partial \mathcal{L}^{\prime}}{\partial \dot{n}}= & a m \\
\frac{\partial \mathcal{L}^{\prime}}{\partial(\boldsymbol{\nabla} n)}= & a m \boldsymbol{u}
\end{aligned}\right.
$$

Taking into account the constraints

$$
\frac{d s}{d t}=0, \frac{d \boldsymbol{r}_{0}}{d t}=0
$$

the Euler-Lagrange equation for $n$ gives

$$
\begin{aligned}
m \frac{d a}{d t}=-\frac{m c^{2}}{\gamma}-\frac{k_{B} T}{\gamma_{A}-1}-n \frac{\partial}{\partial n}\left(\frac{k_{B} T}{\gamma_{A}-1}\right)_{\gamma, s}-m \phi_{g}+m \boldsymbol{u} \cdot \boldsymbol{A}_{g} \\
(\boldsymbol{v}) \delta s\left\{\begin{aligned}
\frac{\partial \mathcal{L}^{\prime}}{\partial s} & =-n \frac{\partial}{\partial s}\left(\frac{k_{B} T}{\gamma_{A}-1}\right)_{n, \gamma} \\
\frac{\partial \mathcal{L}^{\prime}}{\partial \dot{s}} & =b n m \\
\frac{\partial \mathcal{L}^{\prime}}{\partial(\boldsymbol{\nabla} s)} & =b n m \boldsymbol{u}
\end{aligned}\right.
\end{aligned}
$$

The Euler-Lagrange equation for $s$ gives

$$
m \frac{d b}{d t}=-\frac{\partial}{\partial s}\left(\frac{k_{B} T}{\gamma_{A}-1}\right)_{n, \gamma}
$$




$$
(\boldsymbol{v i}) \delta \boldsymbol{r}_{0}\left\{\begin{aligned}
\frac{\partial \mathcal{L}^{\prime}}{\partial \boldsymbol{r}_{0}} & =0 \\
\frac{\partial \mathcal{L}^{\prime}}{\partial \dot{\boldsymbol{r}_{0}}} & =n m \mathbf{c} \\
\frac{\partial \mathcal{L}^{\prime}}{\partial\left(\boldsymbol{\nabla} \boldsymbol{r}_{0}\right)} & =n m \boldsymbol{u c}
\end{aligned}\right.
$$

Finally, the Euler-Lagrange equation for $\boldsymbol{r}_{0}$ gives

$$
\frac{d \mathbf{c}}{d t}=0
$$

The Euler-Lagrange equations $(\boldsymbol{i v}),(\boldsymbol{v})$ and $(\boldsymbol{v} \boldsymbol{i})$ are equations of motion for the Lagrange multipliers $a, b$ and $\mathbf{c}$. These equations must be used to eliminate the Lagrange multipliers from equation (iii) in the form (36). With this objective in mind, consider the following vector and dyadic relations applied to the convective derivatives:

$$
\begin{aligned}
& \frac{d}{d t}(\nabla a)=\frac{\partial}{\partial t} \nabla a+\boldsymbol{u} \cdot \nabla \nabla a \\
& =\boldsymbol{\nabla} \frac{\partial a}{\partial t}+\nabla(\boldsymbol{u} \cdot \nabla a)-\nabla \boldsymbol{u} \cdot(\nabla a)=\nabla \frac{d a}{d t}-\nabla \boldsymbol{u} \cdot(\nabla a), \\
& \frac{d}{d t}(b \boldsymbol{\nabla} s)=\frac{d b}{d t}(\boldsymbol{\nabla} s)+b\left(\frac{\partial}{\partial t} \boldsymbol{\nabla} s+\boldsymbol{u} \cdot \boldsymbol{\nabla} \nabla s\right) \\
& =(\nabla s) \frac{d b}{d t}+b\left(\boldsymbol{\nabla} \frac{\partial s}{\partial t}+\nabla(\boldsymbol{u} \cdot \boldsymbol{\nabla} s)-\boldsymbol{\nabla} \boldsymbol{u} \cdot(\boldsymbol{\nabla} s)\right) \\
& =(\nabla s) \frac{d b}{d t}+b \boldsymbol{\nabla} \frac{d s}{d t}-\nabla \boldsymbol{u} \cdot(b \boldsymbol{\nabla} s), \\
& \frac{d}{d t}\left[\left(\boldsymbol{\nabla} \boldsymbol{r}_{0}\right) \cdot \mathbf{c}\right]=\left(\boldsymbol{\nabla} \boldsymbol{r}_{0}\right) \cdot \frac{d \mathbf{c}}{d t}+\left(\frac{\partial}{\partial t} \boldsymbol{\nabla} \boldsymbol{r}_{0}+\boldsymbol{u} \cdot \nabla \boldsymbol{\nabla} \boldsymbol{r}_{0}\right) \cdot \mathbf{c} \\
& =\left(\boldsymbol{\nabla} \boldsymbol{r}_{0}\right) \cdot \frac{d \mathbf{c}}{d t}+\left(\boldsymbol{\nabla} \frac{\partial \boldsymbol{r}_{0}}{\partial t}+\boldsymbol{\nabla}\left(\boldsymbol{u} \cdot \boldsymbol{\nabla} \boldsymbol{r}_{0}\right)\right) \cdot \mathbf{c} \\
& -\left(\boldsymbol{\nabla} \boldsymbol{r}_{0}\right) \cdot(\boldsymbol{\nabla u}) \cdot \mathbf{c}-\left(\boldsymbol{\nabla} \boldsymbol{r}_{0}\right) \times(\boldsymbol{\nabla} \times \boldsymbol{u}) \cdot \mathbf{c} \\
& =\left(\nabla \boldsymbol{r}_{0}\right) \cdot \frac{d \mathbf{c}}{d t}+\left(\nabla \frac{d \boldsymbol{r}_{0}}{d t}\right) \cdot \mathbf{c} \\
& -(\boldsymbol{\nabla} \boldsymbol{u}) \cdot\left(\boldsymbol{\nabla} \boldsymbol{r}_{0}\right) \cdot \mathbf{c}+\mathbf{c} \times\left[\boldsymbol{\nabla} \times\left(\boldsymbol{u} \cdot \boldsymbol{\nabla} \boldsymbol{r}_{0}\right)\right] \text {. }
\end{aligned}
$$

Using the constraints $d s / d t=d \boldsymbol{r}_{0} / d t=0$ and the Euler-Lagrange equation $(\boldsymbol{v} \boldsymbol{i})$, that is $d \mathbf{c} / d t=0$, the above relations become

$$
\begin{aligned}
\frac{d}{d t}(\nabla a) & =\boldsymbol{\nabla} \frac{d a}{d t}-\boldsymbol{\nabla} \boldsymbol{u} \cdot(\boldsymbol{\nabla} a), \\
\frac{d}{d t}(b \boldsymbol{\nabla} s) & =(\boldsymbol{\nabla} s) \frac{d b}{d t}-\boldsymbol{\nabla} \boldsymbol{u} \cdot(b \boldsymbol{\nabla} s), \\
\frac{d}{d t}\left[\left(\boldsymbol{\nabla} \boldsymbol{r}_{0}\right) \cdot \mathbf{c}\right] & =-(\boldsymbol{\nabla u}) \cdot\left(\boldsymbol{\nabla} \boldsymbol{r}_{0}\right) \cdot \mathbf{c}+\mathbf{c} \times\left[\boldsymbol{\nabla} \times\left(\boldsymbol{u} \cdot \boldsymbol{\nabla} \boldsymbol{r}_{0}\right)\right] .
\end{aligned}
$$

Taking into account the constraint $d \boldsymbol{r}_{0} / d t=0$ it follows that

$$
\frac{d \boldsymbol{r}_{0}}{d t}=\frac{\partial \boldsymbol{r}_{0}}{\partial t}+\boldsymbol{u} \cdot \nabla \boldsymbol{r}_{0}=0 \Longrightarrow \boldsymbol{u} \cdot \boldsymbol{\nabla} \boldsymbol{r}_{0}=-\frac{\partial \boldsymbol{r}_{0}}{\partial t}
$$

Hence

$$
\frac{d}{d t}\left[\left(\boldsymbol{\nabla} \boldsymbol{r}_{0}\right) \cdot \mathbf{c}\right]=-(\boldsymbol{\nabla} \boldsymbol{u}) \cdot\left(\boldsymbol{\nabla} \boldsymbol{r}_{0}\right) \cdot \mathbf{c}-\mathbf{c} \times \frac{\partial}{\partial t} \underbrace{\left(\boldsymbol{\nabla} \times \boldsymbol{r}_{0}\right)}_{0}=-(\boldsymbol{\nabla u}) \cdot\left(\boldsymbol{\nabla} \boldsymbol{r}_{0}\right) \cdot \mathbf{c} .
$$

The previous relations can be collected in the form:

$$
\left\{\begin{aligned}
\frac{d}{d t}(\nabla a) & =\boldsymbol{\nabla} \frac{d a}{d t}-\boldsymbol{\nabla u} \cdot(\boldsymbol{\nabla} a) \\
\frac{d}{d t}(b \boldsymbol{\nabla} s) & =(\boldsymbol{\nabla} s) \frac{d b}{d t}-\boldsymbol{\nabla} \boldsymbol{u} \cdot(b \boldsymbol{\nabla} s) \\
\frac{d}{d t}\left[\left(\boldsymbol{\nabla} \boldsymbol{r}_{0}\right) \cdot \mathbf{c}\right] & =-(\boldsymbol{\nabla u}) \cdot\left(\boldsymbol{\nabla} \boldsymbol{r}_{0}\right) \cdot \mathbf{c}
\end{aligned}\right.
$$


Now, the total time derivative of the Euler-Lagrange equation (36) gives

$$
\begin{aligned}
& \frac{d}{d t}\left(\alpha \boldsymbol{u}+\boldsymbol{A}_{g}\right)=\frac{d}{d t}\left[\boldsymbol{\nabla} a-b \boldsymbol{\nabla} s-\left(\boldsymbol{\nabla} \boldsymbol{r}_{0}\right) \cdot \mathbf{c}\right] \\
& =\boldsymbol{\nabla} \frac{d a}{d t}-\boldsymbol{\nabla u} \cdot(\boldsymbol{\nabla} a)-(\boldsymbol{\nabla} s) \frac{d b}{d t}+\boldsymbol{\nabla} \boldsymbol{u} \cdot(b \boldsymbol{\nabla} s)+(\boldsymbol{\nabla u}) \cdot\left(\boldsymbol{\nabla} \boldsymbol{r}_{0}\right) \cdot \mathbf{c},
\end{aligned}
$$

and the scalar pre-multiplication by $\boldsymbol{\nabla} \boldsymbol{u}$ gives

$$
\boldsymbol{\nabla} \boldsymbol{u} \cdot\left(\alpha \boldsymbol{u}+\boldsymbol{A}_{g}\right)=\boldsymbol{\nabla} \boldsymbol{u} \cdot\left[\boldsymbol{\nabla} a-b \boldsymbol{\nabla} s-\left(\boldsymbol{\nabla} \boldsymbol{r}_{0}\right) \cdot \mathbf{c}\right] .
$$

The addition of these two equations yields

$$
\frac{d}{d t}\left(\alpha \boldsymbol{u}+\boldsymbol{A}_{g}\right)+\nabla \boldsymbol{u} \cdot\left(\alpha \boldsymbol{u}+\boldsymbol{A}_{g}\right)=\nabla \frac{d a}{d t}-(\nabla s) \frac{d b}{d t} .
$$

Finally, using equations $(\boldsymbol{i v})$ and $(\boldsymbol{v})$, the remaining Lagrange multipliers $a$ and $b$ are eliminated from the equation of specific momentum conservation

$$
\begin{aligned}
& \frac{d}{d t}\left(\alpha \boldsymbol{u}+\boldsymbol{A}_{g}\right)+\boldsymbol{\nabla} \boldsymbol{u} \cdot\left(\alpha \boldsymbol{u}+\boldsymbol{A}_{g}\right) \\
& =-\boldsymbol{\nabla} \phi_{g}+\boldsymbol{\nabla}\left(\boldsymbol{u} \cdot \boldsymbol{A}_{g}\right)-c^{2} \boldsymbol{\nabla} \gamma^{-1}-\boldsymbol{\nabla}\left(\frac{k_{B} T / m}{\gamma_{A}-1}\right) \\
& -\boldsymbol{\nabla}\left[n \frac{\partial}{\partial n}\left(\frac{k_{B} T / m}{\gamma_{A}-1}\right)_{\gamma, s}\right]+(\boldsymbol{\nabla} s) \frac{\partial}{\partial s}\left(\frac{k_{B} T / m}{\gamma_{A}-1}\right)_{n, \gamma} .
\end{aligned}
$$

Maxwell's differential relations can be determined from the second law of thermodynamics (25). Thus

$$
\left\{\begin{array}{l}
\frac{\partial}{\partial n}\left(\frac{k_{B} T}{\gamma_{A}-1}\right)_{\gamma, s}=\frac{k_{B} T}{n} \\
\frac{\partial}{\partial \gamma}\left(\frac{k_{B} T}{\gamma_{A}-1}\right)_{n, s}=-\frac{\gamma_{A}}{\gamma_{A}-1} \frac{k_{B} T}{\gamma} \\
\frac{\partial}{\partial s}\left(\frac{k_{B} T}{\gamma_{A}-1}\right)_{n, \gamma}=T
\end{array}\right.
$$

These relations reduce the equation of specific momentum conservation to the form

$$
\begin{aligned}
\frac{d}{d t}\left(\alpha \boldsymbol{u}+\boldsymbol{A}_{g}\right)+\boldsymbol{\nabla u} \cdot\left(\alpha \boldsymbol{u}+\boldsymbol{A}_{g}\right)= & -\boldsymbol{\nabla} \phi_{g}+\boldsymbol{\nabla}\left(\boldsymbol{u} \cdot \boldsymbol{A}_{g}\right)-c^{2} \boldsymbol{\nabla} \gamma^{-1} \\
& -\boldsymbol{\nabla}\left(\frac{k_{B} T}{m}\right)+\frac{k_{B} T}{m}\left(\frac{\gamma_{A}}{\gamma_{A}-1} \frac{\boldsymbol{\nabla} \gamma}{\gamma}-\frac{\boldsymbol{\nabla} n}{n}\right),
\end{aligned}
$$

where

$$
\alpha=\gamma\left(1+\frac{\gamma_{A}}{\gamma_{A}-1} \frac{\gamma k_{B} T}{m c^{2}}\right)
$$

Using

$$
\nabla \gamma^{-1}=-\gamma \frac{\nabla u^{2}}{2 c^{2}} \text { and } \nabla \gamma=\gamma^{3} \frac{\nabla u^{2}}{2 c^{2}}
$$

the specific momentum conservation equation becomes

$$
\frac{d}{d t}(\alpha \boldsymbol{u})=-\frac{\boldsymbol{\nabla}\left(n k_{B} T\right)}{n m}-\boldsymbol{\nabla} \phi_{g}-\frac{\partial \boldsymbol{A}_{g}}{\partial t}-(\boldsymbol{u} \cdot \boldsymbol{\nabla}) \boldsymbol{A}_{g}-(\boldsymbol{\nabla u}) \cdot \boldsymbol{A}_{g}+\nabla\left(\boldsymbol{u} \cdot \boldsymbol{A}_{g}\right) .
$$

Now, the vector and dyadic relations

$$
\left\{\begin{array}{l}
(\boldsymbol{u} \cdot \boldsymbol{\nabla}) \boldsymbol{A}_{g}=\left(\boldsymbol{\nabla} \boldsymbol{A}_{g}\right) \cdot \boldsymbol{u}-\boldsymbol{u} \times\left(\boldsymbol{\nabla} \times \boldsymbol{A}_{g}\right) \\
\boldsymbol{\nabla}\left(\boldsymbol{u} \cdot \boldsymbol{A}_{g}\right)=(\boldsymbol{\nabla u}) \cdot \boldsymbol{A}_{g}+\left(\boldsymbol{\nabla} \boldsymbol{A}_{g}\right) \cdot \boldsymbol{u}
\end{array}\right.
$$


give

$$
\frac{d}{d t}(\alpha \boldsymbol{u})=\underbrace{-\frac{\boldsymbol{\nabla}\left(n k_{B} T\right)}{m n}-\boldsymbol{\nabla} \phi_{g}-\frac{\partial \boldsymbol{A}_{g}}{\partial t}}_{-(\boldsymbol{\nabla} p) / \rho}+\boldsymbol{u} \times \underbrace{\left(\boldsymbol{\nabla} \times \boldsymbol{A}_{g}\right)}_{\boldsymbol{E}_{g}},
$$

where $\boldsymbol{E}_{g}=-\boldsymbol{\nabla} \phi_{g}-\partial \boldsymbol{A}_{g} / \partial t$ and $\boldsymbol{B}_{g}=\boldsymbol{\nabla} \times \boldsymbol{A}_{g}$ are the GE and GM field components, respectively. The equation of specific momentum conservation becomes

$$
\frac{d}{d t}(\alpha \boldsymbol{u})=-\frac{\nabla p}{\rho}+\boldsymbol{E}_{g}+\boldsymbol{u} \times \boldsymbol{B}_{g},
$$

which has the form of a Lorentz acceleration acting on the fluid element. Note that the thermal motion increases the fluid inertia. The relativistic effects introduced by the coefficient $\alpha$ affect the reconnection process in astrophysical plasmas [27] and equally affect the evolution of relativistic fluids. Without the inertial effects included in $\alpha$ (taking $\alpha \rightarrow 1)$, and neglecting the pressure effects for a fluid element $(p \rightarrow 0)$, this equation corresponds to the equation of motion obtained by Thirring in the first approximation to Einstein's gravity equations [12, 13].

Multiplying by $\rho=m n$ and applying the continuity condition $\partial n / \partial t=-\boldsymbol{\nabla} \cdot(n \boldsymbol{u})$ the equation of momentum conservation yields

$$
\frac{\partial}{\partial t}(m n \alpha \boldsymbol{u})+\underbrace{m \alpha \boldsymbol{u} \boldsymbol{\nabla} \cdot(n \boldsymbol{u})+m n \boldsymbol{u} \cdot \boldsymbol{\nabla}(\alpha \boldsymbol{u})}_{\boldsymbol{\nabla} \cdot(m n \alpha \boldsymbol{u} u)}=-\nabla p+m n\left(\boldsymbol{E}_{g}+\boldsymbol{u} \times \boldsymbol{B}_{g}\right) .
$$

Therefore, the full equation of momentum density conservation for a relativistic perfect fluid in the presence of a gravitoelectromagnetic field can be written as

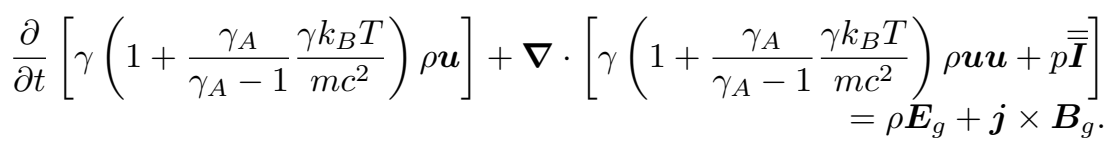

Using the definition (6) of the fluid energy density in the rest frame, the second law (23) becomes

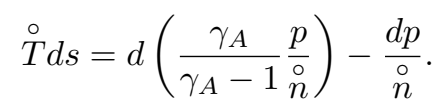

Assuming isentropic flow with $d s=0$ this leads to the differential equation of state

$$
\frac{d p}{\stackrel{\circ}{n}}=d\left(\frac{\gamma_{A}}{\gamma_{A}-1} k_{B} \stackrel{\circ}{T}\right) \text {, }
$$

where $\stackrel{\circ}{n}=n / \gamma$ and $\stackrel{\circ}{T}=\gamma T$. Thus

$$
\frac{\nabla p}{\rho}=\frac{1}{\gamma} \nabla\left(\frac{\gamma_{A}}{\gamma_{A}-1} \frac{\gamma k_{B} T}{m}\right)
$$

can be used to express the fluid pressure in terms of the temperature:

$$
\frac{d}{d t}\left[\gamma\left(1+\frac{\gamma_{A}}{\gamma_{A}-1} \frac{\gamma k_{B} T}{m c^{2}}\right) \boldsymbol{u}\right]=-\frac{1}{\gamma} \nabla\left(\frac{\gamma_{A}}{\gamma_{A}-1} \frac{\gamma k_{B} T}{m}\right)+\boldsymbol{E}_{g}+\boldsymbol{u} \times \boldsymbol{B}_{g} .
$$

The acceleration in this equation can be written in compact form in terms of the function $\alpha$

$$
\frac{d}{d t}(\alpha \boldsymbol{u})=-\frac{c^{2}}{\gamma} \nabla\left(\frac{\alpha}{\gamma}\right)+\boldsymbol{E}_{g}+\boldsymbol{u} \times \boldsymbol{B}_{g}
$$

Scalar multiplication by $\boldsymbol{u}$ and insertion of the Lorentz factor relation $d u^{2}=2 c^{2} d \gamma / \gamma^{3}$ gives

$$
\frac{d}{d t}\left(\alpha c^{2}\right)=\frac{1}{\gamma} \frac{\partial}{\partial t}\left(\frac{\alpha c^{2}}{\gamma}\right)+\boldsymbol{u} \cdot \boldsymbol{E}_{g}
$$


Multiplication by the mass density $\mathrm{nm}$ and use of the continuity condition yields

$$
\frac{\partial}{\partial t}\left(n m c^{2} \alpha\right)+\nabla \cdot\left(n m c^{2} \alpha \boldsymbol{u}\right)=\frac{n m c^{2}}{\gamma} \frac{\partial}{\partial t}\left(\frac{\alpha}{\gamma}\right)+\boldsymbol{j} \cdot \boldsymbol{E}_{g}
$$

Since

$$
d\left(\frac{\alpha}{\gamma}\right)=d\left(\frac{\gamma_{A}}{\gamma_{A}-1} \frac{\gamma k_{B} T}{m c^{2}}\right)=\frac{\gamma d p}{n m c^{2}}
$$

the energy density conservation equation becomes

$$
\frac{\partial}{\partial t}\left(n m c^{2} \alpha\right)+\nabla \cdot\left(n m c^{2} \alpha \boldsymbol{u}\right)=\frac{\partial p}{\partial t}+\boldsymbol{j} \cdot \boldsymbol{E}_{g}
$$

With some rearrangement the energy density conservation equation is written as

$$
\frac{\partial}{\partial t}\left[\gamma \rho c^{2}+\left(\frac{1}{\gamma_{A}-1}+\beta^{2}\right) \gamma^{2} p\right]+\nabla \cdot\left[\left(\gamma \rho c^{2}+\frac{\gamma_{A}}{\gamma_{A}-1} \gamma^{2} p\right) \boldsymbol{u}\right]=\boldsymbol{j} \cdot \boldsymbol{E}_{g}
$$

Summary: The variational procedure demonstrates that fluid flow under the action of a gravitoelectromagnetic field in flat space is governed by the specific momentum conservation equation

$$
\frac{d}{d t}\left[\gamma\left(1+\frac{\gamma_{A}}{\gamma_{A}-1} \frac{\gamma k_{B} T}{m c^{2}}\right) \boldsymbol{u}\right]=-\frac{\nabla p}{\rho}+\boldsymbol{E}_{g}+\boldsymbol{u} \times \boldsymbol{B}_{g},
$$

combined with the equation of continuity (conservation of the number of particles)

$$
\frac{\partial \rho}{\partial t}+\nabla \cdot(\rho \boldsymbol{u})=0
$$

and Maxwell's source equations

$$
\begin{aligned}
\nabla \cdot \boldsymbol{E}_{g} & =-4 \pi G \rho & & \text { GE Gauss's law } \\
\boldsymbol{\nabla} \times \boldsymbol{B}_{g} & =-\frac{4 \pi G}{c^{2}} \rho \boldsymbol{u}+\frac{1}{c^{2}} \frac{\partial \boldsymbol{E}_{g}}{\partial t} & & \text { GEM Ampère's law }
\end{aligned}
$$

The GEM field variables are related to the potentials by

$$
\begin{aligned}
& \boldsymbol{E}_{g}=-\boldsymbol{\nabla} \phi_{g}-\frac{\partial \boldsymbol{A}_{g}}{\partial t}, \\
& \boldsymbol{B}_{g}=\boldsymbol{\nabla} \times \boldsymbol{A}_{g},
\end{aligned}
$$

which leads to the consistency relations

$$
\begin{aligned}
\boldsymbol{\nabla} \times \boldsymbol{E}_{g} & =-\frac{\partial \boldsymbol{B}_{g}}{\partial t} & & \text { GEM Faraday's law } \\
\boldsymbol{\nabla} \cdot \boldsymbol{B}_{g} & =0 & & \text { GM Gauss' law }
\end{aligned}
$$

The pressure $p$ is related to the temperature $T$ according to the isentropic flow condition $d s / d t=0$ (conservation of energy). The equation of state for a perfect fluid, $p=n k_{B} T$, can be used to relate the density $n$ to the temperature $T$.

Introducing the weakly relativistic approximation, $\gamma \sim 1+u^{2} / 2 c^{2}$, the energy density conservation equation (74) reduces to

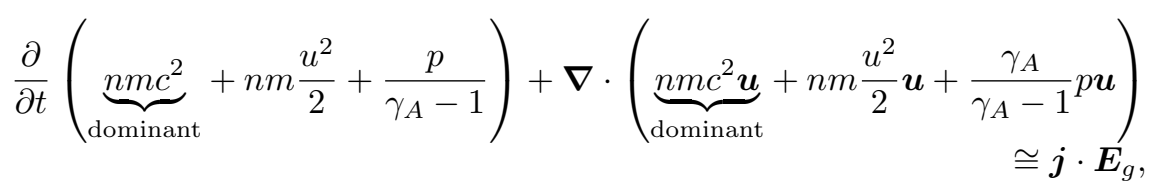


where the dominant terms (in the expansion in powers of $1 / c$ ) cancel due to fluid continuity. Similarly, the equation of momentum density conservation reduces to

$$
\frac{\partial}{\partial t}(n m \boldsymbol{u})+\nabla \cdot(n m \boldsymbol{u} \boldsymbol{u}+p \overline{\overline{\boldsymbol{I}}}) \cong \rho \boldsymbol{E}_{g}+\boldsymbol{j} \times \boldsymbol{B}_{g}
$$

In the weak relativistic approximation the fluid motion is described by the equations of continuity, specific momentum conservation (in the equivalent Lorentz acceleration form), the laws of Gauss and Ampère, and the adiabatic equation of state with $\gamma_{A} \cong 5 / 3$ for a monatomic gas, which is equivalent to the energy conservation equation:

$$
\left\{\begin{aligned}
\frac{\partial \rho}{\partial t}+\nabla \cdot(\rho \boldsymbol{u}) & =0 \\
\frac{d \boldsymbol{u}}{d t} & \cong-\boldsymbol{\nabla}\left(\frac{\gamma_{A}}{\gamma_{A}-1} \frac{p}{\rho}\right)+\boldsymbol{E}_{g}+\boldsymbol{u} \times \boldsymbol{B}_{g} \\
\boldsymbol{\nabla} \cdot \boldsymbol{E}_{g} & =-4 \pi G \rho \\
\boldsymbol{\nabla} \times \boldsymbol{B}_{g} & =-\frac{4 \pi G}{c^{2}} \rho \boldsymbol{u}+\frac{1}{c^{2}} \frac{\partial \boldsymbol{E}_{g}}{\partial t} \\
p & \propto \rho^{\gamma_{A}}
\end{aligned}\right.
$$

The gravitoelectric and gravitomagnetic fields $\boldsymbol{E}_{g}$ and $\boldsymbol{B}_{g}$ are given in terms of the potentials $\phi_{g}$ and $\boldsymbol{A}_{g}$, characterizing a system of 9 equations in 9 unknowns. The equations of motion in the weak relativistic form can be used to describe, for example, the initial evolution of galactic systems. In particular, a dust distribution is governed by the above set of equations taking $p \sim 0[18]$.

\section{B. Lagrangian variational principle}

In this subsection the equations of fluid motion are considered within the Lagrangian approach. Changing from Eulerian $(\boldsymbol{r}, t)$ to Lagrangian $\left(\boldsymbol{r}_{0}, \tau\right)$ coordinates, Hamilton's principle becomes

$$
\delta \int d^{3} r_{0} d \tau J\left(-\frac{n m c^{2}}{\gamma}-\frac{n k_{B} T}{\gamma_{A}-1}-n m \phi_{g}+n m \boldsymbol{A}_{g} \cdot \frac{\partial \boldsymbol{\xi}}{\partial \tau}-\frac{E_{g}^{2}-c^{2} B_{g}^{2}}{8 \pi G}\right)=0
$$

$\overline{\bar{J}}$ is the Jacobian dyadic

$$
\overline{\bar{J}}=\nabla_{0} \boldsymbol{r}=\nabla_{0}\left(\boldsymbol{r}_{0}+\boldsymbol{\xi}\right)=\overline{\overline{\boldsymbol{I}}}+\nabla_{0} \boldsymbol{\xi}
$$

and $J=|\overline{\bar{J}}|$ its determinant. In this variational principle the integration extends over the fixed initial positions and the field variables are considered as functions of $\boldsymbol{r}_{0}$ and $\tau$ (these coordinates may be considered as fixed on the fluid element). The velocity of a fluid element is given in terms of the Lagrangian displacement $\boldsymbol{\xi}=\boldsymbol{r}-\boldsymbol{r}_{0}$ by $\boldsymbol{u}=\partial \boldsymbol{\xi} / \partial \tau$ (the velocity is defined at the position of the fluid element, which may be considered as a particle) and the Lagrangian form of the equation of continuity is ( $J$ represents the dilatation of an infinitesimal fluid volume as it follows the motion [19])

$$
n J=n_{0}\left(\boldsymbol{r}_{0}, \tau=0\right) .
$$

The equation of conservation of entropy becomes

$$
\partial s / \partial \tau=0
$$

Introducing the Lagrange multipliers $a_{L}$ and $b_{L}$ :

$$
\begin{array}{r}
\delta \int d^{3} r_{0} d \tau\left\{J\left[-\frac{n m c^{2}}{\gamma}-\frac{n k_{B} T}{\gamma_{A}-1}-n m \phi_{g}+n m \boldsymbol{A}_{g} \cdot \frac{\partial \boldsymbol{\xi}}{\partial \tau}-\frac{E_{g}^{2}-c^{2} B_{g}^{2}}{8 \pi G}\right]\right. \\
\left.+\left[a_{L}\left(n J-n_{0}\right)+b_{L} \frac{\partial s}{\partial \tau}\right]\right\}=0
\end{array}
$$


The Euler-Lagrange equations for the field variables $\varphi=\left(\phi_{g}, \boldsymbol{A}_{g}, \boldsymbol{\xi}, n, s\right)$ are:

$$
(\boldsymbol{i}) \delta \phi_{g}\left\{\begin{array}{l}
\frac{\partial \mathcal{L}_{0}^{\prime}}{\partial \phi_{g}}=-J n m=-n_{0} m=-\rho_{0} \\
\frac{\partial \mathcal{L}_{0}^{\prime}}{\partial\left(\partial \phi_{g} / \partial \tau\right)}=0 \\
\frac{\partial \mathcal{L}_{0}^{\prime}}{\partial\left(\nabla_{0} \phi_{g}\right)}=\frac{\boldsymbol{E}_{g}}{4 \pi G}
\end{array}\right.
$$

The Euler-Lagrange equation for $\phi_{g}$ gives the GE Gauss' law in Lagrangian coordinates

$$
\begin{gathered}
\boldsymbol{\nabla}_{0} \cdot \boldsymbol{E}_{g}=-4 \pi G \rho_{0} . \\
(\boldsymbol{i i}) \delta \boldsymbol{A}_{g}\left\{\begin{array}{l}
\frac{\partial \mathcal{L}_{0}^{\prime}}{\partial \boldsymbol{A}_{g}}=J n m \frac{\partial \boldsymbol{\xi}}{\partial \tau}=n_{0} m \frac{\partial \boldsymbol{\xi}}{\partial \tau}=\boldsymbol{j}_{0} \\
\frac{\partial \mathcal{L}_{0}^{\prime}}{\partial\left(\partial \boldsymbol{A}_{g} / \partial \tau\right)}=\frac{\boldsymbol{E}_{g}}{4 \pi G} \\
\frac{\partial \mathcal{L}_{0}^{\prime}}{\partial\left(\boldsymbol{\nabla}_{0} \boldsymbol{A}_{g}\right)}=-\frac{c^{2} \boldsymbol{B}_{g}}{4 \pi G} \cdot \overline{\overline{\boldsymbol{\epsilon}}}
\end{array}\right.
\end{gathered}
$$

The Euler-Lagrange equation for $\boldsymbol{A}_{g}$ gives the GEM Ampère's law in Lagrangian coordinates

$$
\begin{aligned}
& \nabla_{0} \times \boldsymbol{B}_{g}=-\frac{4 \pi G}{c^{2}} \boldsymbol{j}_{0}+\frac{1}{c^{2}} \frac{\partial \boldsymbol{E}_{g}}{\partial \tau} . \\
& (\boldsymbol{i i i}) \delta \boldsymbol{\xi}\left\{\begin{array}{c}
\frac{\partial \mathcal{L}_{0}^{\prime}}{\partial \boldsymbol{\xi}}=J n m\left[-\frac{\partial \phi_{g}}{\partial \boldsymbol{\xi}}+\left(\frac{\partial \boldsymbol{A}_{g}}{\partial \boldsymbol{\xi}}\right) \cdot \frac{\partial \boldsymbol{\xi}}{\partial \tau}\right]=J n m\left[-\boldsymbol{\nabla} \phi_{g}+\left(\boldsymbol{\nabla} \boldsymbol{A}_{g}\right) \cdot \frac{\partial \boldsymbol{\xi}}{\partial \tau}\right] \\
\frac{\partial \mathcal{L}_{0}^{\prime}}{\partial(\partial \boldsymbol{\xi} / \partial \tau)}=J\left[n \gamma m c^{2}-n \gamma^{3} \frac{\partial}{\partial \gamma}\left(\frac{k_{B} T}{\gamma_{A}-1}\right)_{n, s}\right] \frac{1}{c^{2}} \frac{\partial \boldsymbol{\xi}}{\partial \tau}+J n m \boldsymbol{A}_{g} \\
\frac{\partial \mathcal{L}_{0}^{\prime}}{\partial\left(\boldsymbol{\nabla}_{0} \boldsymbol{\xi}\right)}=\frac{\partial J}{\partial\left(\boldsymbol{\nabla}_{0} \boldsymbol{r}\right)}\left(-\frac{n m c^{2}}{\gamma}-\frac{n k_{B} T}{\gamma_{A}-1}\right. \\
\left.-n m \phi_{g}+n m \boldsymbol{A}_{g} \cdot \frac{\partial \boldsymbol{\xi}}{\partial \tau}+a_{L} n\right)
\end{array}\right.
\end{aligned}
$$

The Euler-Lagrange equation for $\boldsymbol{\xi}$ gives

$$
\begin{aligned}
& \text { Jnm }\left[-\frac{\partial \phi_{g}}{\partial \boldsymbol{\xi}}+\left(\frac{\partial \boldsymbol{A}_{g}}{\partial \boldsymbol{\xi}}\right) \cdot \frac{\partial \boldsymbol{\xi}}{\partial \tau}\right] \\
& =\frac{\partial}{\partial \tau}\left\{J\left[n \gamma m c^{2}-n \gamma^{3} \frac{\partial}{\partial \gamma}\left(\frac{k_{B} T}{\gamma_{A}-1}\right)_{n, s}\right] \frac{1}{c^{2}} \frac{\partial \boldsymbol{\xi}}{\partial \tau}+J n m \boldsymbol{A}_{g}\right\} \\
& +\boldsymbol{\nabla}_{0} \cdot\left[\frac{\partial J}{\partial\left(\boldsymbol{\nabla}_{0} \boldsymbol{r}\right)}\left(-\frac{n m c^{2}}{\gamma}-\frac{n k_{B} T}{\gamma_{A}-1}-n m \phi_{g}+n m \boldsymbol{A}_{g} \cdot \frac{\partial \boldsymbol{\xi}}{\partial \tau}+a_{L} n\right)\right] . \\
& (\boldsymbol{i v}) \delta n\left\{\begin{array}{l}
\frac{\partial \mathcal{L}_{0}^{\prime}}{\partial n}=J\left[-\frac{m c^{2}}{\gamma}-\frac{k_{B} T}{\gamma_{A}-1}-n \frac{\partial}{\partial n}\left(\frac{k_{B} T}{\gamma_{A}-1}\right)_{\gamma, s}\right. \\
\frac{\partial \mathcal{L}_{0}^{\prime}}{\partial(\partial n / \partial \tau)}=0 \\
\frac{\partial \mathcal{L}_{0}^{\prime}}{\partial\left(\boldsymbol{\nabla}_{0} n\right)}=0
\end{array}\right.
\end{aligned}
$$

The Euler-Lagrange equation for $n$ gives the value of the Lagrange multiplier $a_{L}$ ( $J$ must be different from zero so that the transformation $\boldsymbol{r}_{0} \rightleftarrows \boldsymbol{r}$ is one-to-one)

$$
a_{L}=\frac{m c^{2}}{\gamma}+\frac{k_{B} T}{\gamma_{A}-1}+n \frac{\partial}{\partial n}\left(\frac{k_{B} T}{\gamma_{A}-1}\right)_{\gamma, s}+m \phi_{g}-m \boldsymbol{A}_{g} \cdot \frac{\partial \boldsymbol{\xi}}{\partial \tau} .
$$


Finally, the Euler-Lagrange equation for $s$ gives

$$
\frac{\partial b_{L}}{\partial \tau}=-J n \frac{\partial}{\partial s}\left(\frac{k_{B} T}{\gamma_{A}-1}\right)_{n, \gamma} .
$$

Now, the expression $(\boldsymbol{i v})$ for $a_{L}$ can be substituted in the Euler-Lagrange equation $(\boldsymbol{i i i})$ for $\boldsymbol{\xi}$,

$$
\begin{aligned}
& \frac{\partial}{\partial \tau}\left\{J\left[n \gamma m c^{2}-n \gamma^{3} \frac{\partial}{\partial \gamma}\left(\frac{k_{B} T}{\gamma_{A}-1}\right)_{n, s}\right] \frac{1}{c^{2}} \frac{\partial \boldsymbol{\xi}}{\partial \tau}+J n m \boldsymbol{A}_{g}\right\} \\
& +\nabla_{0} \cdot\left[\frac{\partial J}{\partial\left(\boldsymbol{\nabla}_{0} \boldsymbol{r}\right)} n^{2} \frac{\partial}{\partial n}\left(\frac{k_{B} T}{\gamma_{A}-1}\right)_{\gamma, s}\right]=J n m\left[-\frac{\partial \phi_{g}}{\partial \boldsymbol{\xi}}+\left(\frac{\partial \boldsymbol{A}_{g}}{\partial \boldsymbol{\xi}}\right) \cdot \frac{\partial \boldsymbol{\xi}}{\partial \tau}\right] .
\end{aligned}
$$

The derivative of the determinant of a second-order tensor gives

$$
\frac{\partial J}{\partial\left(\boldsymbol{\nabla}_{0} \boldsymbol{r}\right)}=\frac{\partial}{\partial\left(\boldsymbol{\nabla}_{0} \boldsymbol{r}\right)}\left|\boldsymbol{\nabla}_{0} \boldsymbol{r}\right|=\overline{\overline{\boldsymbol{C}}}=J\left[\left(\boldsymbol{\nabla}_{0} \boldsymbol{r}\right)^{-1}\right]^{T},
$$

where $J=\left|\nabla_{0} \boldsymbol{r}\right|$ is the determinant of $\boldsymbol{\nabla}_{0} \boldsymbol{r}$ and $\overline{\bar{C}}$ is the dyadic of the cofactors of the elements of $\boldsymbol{\nabla}_{0} \boldsymbol{r}$. Moreover, the transformation from Eulerian to Lagrangian coordinates gives

$$
\boldsymbol{\nabla}_{0} \equiv\left(\boldsymbol{\nabla}_{0} \boldsymbol{r}\right) \cdot \boldsymbol{\nabla} \equiv \overline{\overline{\boldsymbol{J}}} \cdot \boldsymbol{\nabla} \text { and } \boldsymbol{\nabla} \equiv\left(\boldsymbol{\nabla} \boldsymbol{r}_{0}\right) \cdot \nabla_{0} \equiv \overline{\overline{\boldsymbol{J}}}^{-1} \cdot \boldsymbol{\nabla}_{0} \Rightarrow \boldsymbol{\nabla} \boldsymbol{r}_{0}=\left(\boldsymbol{\nabla}_{0} \boldsymbol{r}\right)^{-1}
$$

Note the identities

$$
\overline{\overline{\boldsymbol{J}}} \cdot \overline{\overline{\boldsymbol{C}}}^{T}=\overline{\overline{\boldsymbol{C}}} \cdot \overline{\overline{\boldsymbol{J}}}^{T}=J \overline{\overline{\boldsymbol{I}}} \text { and } \overline{\overline{\boldsymbol{C}}}=J\left(\overline{\overline{\boldsymbol{J}}}^{-1}\right)^{T}
$$

Thus

$$
\frac{\partial J}{\partial\left(\boldsymbol{\nabla}_{0} \boldsymbol{r}\right)}=J\left(\boldsymbol{\nabla} \boldsymbol{r}_{0}\right)^{T}
$$

It follows that

$$
\begin{aligned}
& \boldsymbol{\nabla}_{0} \cdot\left[J\left(\boldsymbol{\nabla} \boldsymbol{r}_{0}\right)^{T} n^{2} \frac{\partial}{\partial n}\left(\frac{k_{B} T}{\gamma_{A}-1}\right)_{\gamma, s}\right] \\
& =\nabla_{0} \cdot\left[J\left(\boldsymbol{\nabla} \boldsymbol{r}_{0}\right)^{T}\right] n^{2} \frac{\partial}{\partial n}\left(\frac{k_{B} T}{\gamma_{A}-1}\right)_{\gamma, s}+J\left(\boldsymbol{\nabla} \boldsymbol{r}_{0}\right) \cdot \nabla_{0}\left[n^{2} \frac{\partial}{\partial n}\left(\frac{k_{B} T}{\gamma_{A}-1}\right)_{\gamma, s}\right] .
\end{aligned}
$$

But the first term on the right-hand side vanishes, since

$$
\boldsymbol{\nabla}_{0} \cdot\left[J\left(\boldsymbol{\nabla} \boldsymbol{r}_{0}\right)^{T}\right]=\nabla_{0} \cdot \overline{\bar{C}}=0
$$

and the second term can be written as

$$
J\left(\boldsymbol{\nabla} \boldsymbol{r}_{0}\right) \cdot \nabla_{0}\left[n^{2} \frac{\partial}{\partial n}\left(\frac{k_{B} T}{\gamma_{A}-1}\right)_{\gamma, s}\right]=J \boldsymbol{\nabla}\left[n^{2} \frac{\partial}{\partial n}\left(\frac{k_{B} T}{\gamma_{A}-1}\right)_{\gamma, s}\right]
$$

so that the Euler-Lagrange equation (97) for $\boldsymbol{\xi}$ becomes

$$
\begin{aligned}
& \frac{\partial}{\partial \tau}\left\{J\left[n \gamma m c^{2}-n \gamma^{3} \frac{\partial}{\partial \gamma}\left(\frac{k_{B} T}{\gamma_{A}-1}\right)_{n, s}\right] \frac{1}{c^{2}} \frac{\partial \boldsymbol{\xi}}{\partial \tau}+J n m \boldsymbol{A}_{g}\right\} \\
& +J \boldsymbol{\nabla}\left[n^{2} \frac{\partial}{\partial n}\left(\frac{k_{B} T}{\gamma_{A}-1}\right)_{\gamma, s}\right]=J n m\left[-\boldsymbol{\nabla} \phi_{g}+\left(\boldsymbol{\nabla} \boldsymbol{A}_{g}\right) \cdot \frac{\partial \boldsymbol{\xi}}{\partial \tau}\right] .
\end{aligned}
$$


The quantity $n J=n_{0}$ is independent of $\tau$, therefore

$$
\begin{aligned}
& \frac{\partial}{\partial \tau}\left[\gamma \frac{\partial \boldsymbol{\xi}}{\partial \tau}-\frac{\gamma^{3}}{m c^{2}} \frac{\partial}{\partial \gamma}\left(\frac{k_{B} T}{\gamma_{A}-1}\right)_{n, s} \frac{\partial \boldsymbol{\xi}}{\partial \tau}+\boldsymbol{A}_{g}\right] \\
& =-\frac{1}{n m} \nabla\left[n^{2} \frac{\partial}{\partial n}\left(\frac{k_{B} T}{\gamma_{A}-1}\right)_{\gamma, s}\right]-\nabla \phi_{g}+\left(\boldsymbol{\nabla} \boldsymbol{A}_{g}\right) \cdot \frac{\partial \boldsymbol{\xi}}{\partial \tau} .
\end{aligned}
$$

Using the thermodynamic relations (55) the time rate of change of the specific canonical momentum of the fluid element is obtained:

$$
\frac{\partial}{\partial \tau}\left[\gamma\left(1+\frac{\gamma_{A}}{\gamma_{A}-1} \frac{\gamma k_{B} T}{m c^{2}}\right) \frac{\partial \boldsymbol{\xi}}{\partial \tau}+\boldsymbol{A}_{g}\right]=-\frac{\boldsymbol{\nabla} p}{\rho}-\nabla \phi_{g}+\left(\boldsymbol{\nabla} \boldsymbol{A}_{g}\right) \cdot \frac{\partial \boldsymbol{\xi}}{\partial \tau} .
$$

The transformation back to Eulerian coordinates is carried out by means of the relations

$$
\frac{\partial}{\partial \tau} \equiv \frac{\partial}{\partial t}+\boldsymbol{u} \cdot \boldsymbol{\nabla} \text { and } \frac{\partial \boldsymbol{\xi}}{\partial \tau}=\boldsymbol{u}
$$

Hence

$$
\begin{aligned}
& \left(\frac{\partial}{\partial t}+\boldsymbol{u} \cdot \boldsymbol{\nabla}\right)\left[\gamma\left(1+\frac{\gamma_{A}}{\gamma_{A}-1} \frac{\gamma k_{B} T}{m c^{2}}\right) \boldsymbol{u}\right] \\
& =-\frac{\boldsymbol{\nabla} p}{\rho}-\boldsymbol{\nabla} \phi_{g}-\dot{\boldsymbol{A}_{g}}-\boldsymbol{u} \cdot \boldsymbol{\nabla} \boldsymbol{A}_{g}+\left(\boldsymbol{\nabla} \boldsymbol{A}_{g}\right) \cdot \boldsymbol{u} .
\end{aligned}
$$

Multiplying by $\mathrm{nm}$ and using the equation of continuity, this equation becomes

$$
\begin{aligned}
& \frac{\partial}{\partial t}\left(n \gamma m \boldsymbol{u}+\frac{\gamma_{A}}{\gamma_{A}-1} n \gamma^{2} k_{B} T \frac{\boldsymbol{u}}{c^{2}}\right) \\
& +\boldsymbol{\nabla} \cdot\left[n \gamma m \boldsymbol{u} \boldsymbol{u}+n k_{B} T \overline{\overline{\boldsymbol{I}}}+\frac{\gamma_{A}}{\gamma_{A}-1} n \gamma^{2} k_{B} T \frac{\boldsymbol{u} \boldsymbol{u}}{c^{2}}\right] \\
& \quad=-n m \boldsymbol{\nabla} \phi_{g}-n m \dot{\boldsymbol{A}}_{g}+n m \boldsymbol{u} \times\left(\boldsymbol{\nabla} \times \boldsymbol{A}_{g}\right),
\end{aligned}
$$

which is again the equation of motion in Eulerian form. This can be easily verified using the definitions $\rho=n m$, $\boldsymbol{j}=n m \boldsymbol{u}, \boldsymbol{E}_{g}=-\boldsymbol{\nabla} \phi_{g}-\partial \boldsymbol{A}_{g} / \partial t$ and $\boldsymbol{B}_{g}=\boldsymbol{\nabla} \times \boldsymbol{A}_{g}$, and the equation of state $p=n k_{B} T$, which returns equation (64)

\section{Hamiltonian density and energy integral}

The momentum densities $\pi=\partial \mathcal{L} / \partial \dot{\varphi}$ canonically conjugate to the Lagrangian field coordinates $\phi_{g}, \boldsymbol{A}_{g}, \boldsymbol{\xi}, n$ and $s$ are

$$
\left\{\begin{aligned}
\pi_{\phi_{g}} & =0 \\
\boldsymbol{\pi}_{\boldsymbol{A}_{g}} & =\frac{\boldsymbol{E}_{g}}{4 \pi G} \\
\boldsymbol{\pi}_{\boldsymbol{\xi}} & =n_{0} \gamma m\left(1+\frac{\gamma_{A}}{\gamma_{A}-1} \frac{\gamma k_{B} T}{m c^{2}}\right) \frac{\partial \boldsymbol{\xi}}{\partial \tau}+n_{0} m \boldsymbol{A}_{g} \\
\pi_{n} & =0 \\
\pi_{s} & =b
\end{aligned}\right.
$$

Using the Lagragian density

$$
\mathcal{L}_{0}=-\frac{n_{0} m c^{2}}{\gamma}-\frac{n_{0} k_{B} T}{\gamma_{A}-1}-n_{0} m \phi_{g}+n_{0} m \boldsymbol{A}_{g} \cdot \frac{\partial \boldsymbol{\xi}}{\partial \tau}-\frac{E_{g}^{2}-c^{2} B_{g}^{2}}{8 \pi G},
$$


the Hamiltonian density in Lagrangian coordinates can be easily calculated:

$$
\begin{aligned}
\mathcal{H}_{0}= & \sum_{\alpha} \pi_{\alpha} \frac{\partial \varphi_{\alpha}}{\partial \tau}-\mathcal{L}_{0} \\
= & \frac{\boldsymbol{E}_{g}}{4 \pi G} \cdot \frac{\partial \boldsymbol{A}_{g}}{\partial \tau}+n_{0} \gamma m\left(1+\frac{\gamma_{A}}{\gamma_{A}-1} \frac{\gamma k_{B} T}{m c^{2}}\right)\left(\frac{\partial \boldsymbol{\xi}}{\partial \tau}\right)^{2}+n_{0} m \boldsymbol{A}_{g} \cdot \frac{\partial \boldsymbol{\xi}}{\partial \tau} \\
& -\left(-\frac{n_{0} m c^{2}}{\gamma}-\frac{n_{0} k_{B} T}{\gamma_{A}-1}-n_{0} m \phi_{g}+n_{0} m \boldsymbol{A}_{g} \cdot \frac{\partial \boldsymbol{\xi}}{\partial \tau}-\frac{E_{g}^{2}-c^{2} B_{g}^{2}}{8 \pi G}\right) .
\end{aligned}
$$

Hence

$$
\mathcal{H}_{0}=n_{0} \gamma m c^{2}+\left(1+\frac{\gamma_{A} \beta^{2}}{1-\beta^{2}}\right) \frac{n_{0} k_{B} T}{\gamma_{A}-1}+n_{0} m \phi_{g}+\frac{\boldsymbol{E}_{g}}{4 \pi G} \cdot \frac{\partial \boldsymbol{A}_{g}}{\partial \tau}+\frac{E_{g}^{2}-c^{2} B_{g}^{2}}{8 \pi G} .
$$

Recalling that $n_{0}=n J$, the Hamiltonian density can be defined in the Eulerian sense by

$$
\mathcal{H}=\gamma \rho c^{2}+\left(1+\frac{\gamma_{A} \beta^{2}}{1-\beta^{2}}\right) \frac{p}{\gamma_{A}-1}+\rho \phi_{g}+\frac{\boldsymbol{E}_{g}}{4 \pi G} \cdot \dot{\boldsymbol{A}_{g}}+\frac{E_{g}^{2}-c^{2} B_{g}^{2}}{8 \pi G},
$$

so that

$$
H=\int d^{3} r_{0} J \mathcal{H}_{0}=\int d^{3} r \mathcal{H}
$$

Note that, although the same graphical symbol was used, the gravitoelectromagnetic field variables in Eulerian and Lagrangian coordinates correspond to energy densities related by the ratio $n / n_{0}$. The gravitoelectromagnetic field Hamiltonian density can also be written in the form

$$
\frac{\boldsymbol{E}_{g}}{4 \pi G} \cdot \dot{\boldsymbol{A}_{g}}+\frac{E_{g}^{2}-c^{2} B_{g}^{2}}{8 \pi G}=-\frac{\boldsymbol{E}_{g} \cdot \boldsymbol{\nabla} \phi_{g}}{4 \pi G}-\frac{E_{g}^{2}+c^{2} B_{g}^{2}}{8 \pi G} .
$$

Hence, using the GE Gauss's law

$$
\mathcal{H}=\gamma \rho c^{2}+\left(1+\frac{\gamma_{A} \beta^{2}}{1-\beta^{2}}\right) \frac{p}{\gamma_{A}-1}-\frac{1}{4 \pi G} \nabla \cdot\left(\boldsymbol{E}_{g} \phi_{g}\right)-\frac{E_{g}^{2}+c^{2} B_{g}^{2}}{8 \pi G} .
$$

If $F$ is the volume integral of a density function $\mathcal{F}\left(\pi_{\alpha}, \varphi_{\alpha}, \nabla \varphi_{\alpha}\right)$, its time derivative is given by

$$
\frac{d F}{d t}=\frac{\partial F}{\partial t}+\sum_{\alpha} \int\{\mathcal{F}, \mathcal{H}\}_{\alpha} d^{3} r
$$

where

$$
\{\mathcal{F}, \mathcal{H}\}_{\alpha}=\frac{\delta \mathcal{F}}{\delta \varphi_{\alpha}} \frac{\delta \mathcal{H}}{\delta \pi_{\alpha}}-\frac{\delta \mathcal{F}}{\delta \pi_{\alpha}} \frac{\delta \mathcal{H}}{\delta \varphi_{\alpha}}
$$

is the classical Poisson bracket for $\mathcal{F}$ and $\mathcal{H}$ and the functional derivative notation indicates the variation of $\mathcal{H}$ for small differences $\delta \varphi_{\alpha}$ in the path followed by the field variable $\varphi_{\alpha}$ :

$$
\frac{\delta \mathcal{H}}{\delta \varphi_{\alpha}}=\frac{\partial \mathcal{H}}{\partial \varphi_{\alpha}}-\nabla \cdot\left(\frac{\partial \mathcal{H}}{\partial\left(\nabla \varphi_{\alpha}\right)}\right) .
$$

The relation involving the Poisson bracket constitutes the noncanonical Hamiltonian representation of a system. In particular, taking $\mathcal{F}=\mathcal{H}$ leads to the energy integral

$$
\frac{d H}{d t}=\frac{d}{d t} \int \mathcal{H} d^{3} r=0
$$

Hence

$$
\begin{array}{r}
\frac{d H}{d t}=\frac{d}{d t} \int\left[\gamma \rho c^{2}+\left(1+\frac{\gamma_{A} \beta^{2}}{1-\beta^{2}}\right)\right. \\
\left.\frac{p}{\gamma_{A}-1}-\frac{E_{g}^{2}+c^{2} B_{g}^{2}}{8 \pi G}\right] d^{3} r \\
-\frac{d}{d t} \oint\left(\frac{\phi_{g} \boldsymbol{E}_{g}}{4 \pi G}\right) \cdot d^{2} \boldsymbol{r}=0 .
\end{array}
$$

The stability of the fluid mass distribution in the gravitoelectromagnetic field can be investigated introducing a Lagrangian perturbation $\boldsymbol{\xi}$ in the energy integral, which can be reduced to an energy principle. 


\section{A HYDROGRAVITOMAGNETIC CAUCHY INVARIANT}

Making use of the vector relation

$$
(\boldsymbol{A} \cdot \boldsymbol{\nabla}) \boldsymbol{A}=(\boldsymbol{\nabla} \times \boldsymbol{A}) \times \boldsymbol{A}+\frac{1}{2} \nabla(\boldsymbol{A} \cdot \boldsymbol{A})
$$

applied to the vector $\alpha \boldsymbol{u}$, the fluid acceleration can be written in the form

$$
\frac{d}{d t}(\alpha \boldsymbol{u})=\frac{\partial}{\partial t}(\alpha \boldsymbol{u})+\boldsymbol{u} \cdot \nabla(\alpha \boldsymbol{u})=\frac{\partial}{\partial t}(\alpha \boldsymbol{u})+(\nabla \times \alpha \boldsymbol{u}) \times \boldsymbol{u}+\frac{\nabla(\alpha u)^{2}}{2 \alpha},
$$

and the curl gives, using the continuity equation,

$$
\begin{aligned}
& \boldsymbol{\nabla} \times\left(\frac{d}{d t}(\alpha \boldsymbol{u})\right)= \\
& =\frac{\partial}{\partial t}[\boldsymbol{\nabla} \times(\alpha \boldsymbol{u})]+\boldsymbol{\nabla} \times[(\boldsymbol{\nabla} \times \alpha \boldsymbol{u}) \times \boldsymbol{u}]+\left(\frac{\nabla u^{2}}{2}\right) \times \boldsymbol{\nabla} \alpha \\
& =\frac{d}{d t}[\boldsymbol{\nabla} \times(\alpha \boldsymbol{u})]-\frac{(\boldsymbol{\nabla} \times \alpha \boldsymbol{u})}{\rho} \frac{d \rho}{d t}-[(\boldsymbol{\nabla} \times \alpha \boldsymbol{u}) \cdot \boldsymbol{\nabla}] \boldsymbol{u}+\left(\frac{\boldsymbol{\nabla} u^{2}}{2}\right) \times \nabla \alpha .
\end{aligned}
$$

Thus

$$
\frac{d}{d t}\left(\frac{\nabla \times(\alpha \boldsymbol{u})}{\rho}\right)=\left(\frac{\nabla \times(\alpha \boldsymbol{u})}{\rho} \cdot \nabla\right) \boldsymbol{u}+\frac{1}{\rho} \boldsymbol{\nabla} \times\left(\frac{d}{d t}(\alpha \boldsymbol{u})\right)-\frac{\left(\nabla u^{2}\right) \times \nabla \alpha}{2 \rho} .
$$

Using the expression of the relativistic inertia factor

$$
\alpha=\gamma\left(1+\frac{\gamma_{A}}{\gamma_{A}-1} \frac{\gamma k_{B} T}{m c^{2}}\right)
$$

one obtains

$$
\nabla \alpha=\frac{\partial \alpha}{\partial u} \nabla u+\frac{\partial \alpha}{\partial T} \nabla T
$$

where

$$
\left\{\begin{array}{l}
\frac{\partial \alpha}{\partial u}=\left(1+\frac{\gamma_{A}}{\gamma_{A}-1} \frac{2 \gamma k_{B} T}{m c^{2}}\right) \frac{d \gamma}{d u} \\
\frac{\partial \alpha}{\partial T}=\frac{\gamma_{A}}{\gamma_{A}-1} \frac{\gamma^{2} k_{B} T}{m c^{2}}\left(\frac{1}{T}-\frac{d \gamma_{A} / d T}{\gamma_{A}\left(\gamma_{A}-1\right)}\right)
\end{array}\right.
$$

Taking into account the transformation of the absolute temperature $T=\stackrel{\circ}{T} / \gamma$ from the frame moving with the fluid velocity $\boldsymbol{u}$ to the rest frame temperature $\stackrel{\circ}{T}$, the above expression for $\nabla \alpha$ becomes

$$
\nabla \alpha=\left(\frac{\partial \alpha}{\partial u}-\frac{\partial \alpha}{\partial T} \frac{\gamma^{2} v T}{c^{2}}\right) \nabla u \Longrightarrow \nabla u \times \nabla \alpha=0
$$

This leads to a diffusion equation for the vorticity

$$
\frac{d}{d t}\left(\frac{\nabla \times(\alpha \boldsymbol{u})}{\rho}\right)=\left(\frac{\nabla \times(\alpha \boldsymbol{u})}{\rho} \cdot \nabla\right) \boldsymbol{u}+\frac{1}{\rho} \nabla \times\left(\frac{d}{d t}(\alpha \boldsymbol{u})\right) .
$$

Now, the equation of specific momentum conservation

$$
\frac{d}{d t}(\alpha \boldsymbol{u})=-\frac{\nabla p}{\rho}+\boldsymbol{E}_{g}+\boldsymbol{u} \times \boldsymbol{B}_{g}
$$

gives

$$
\boldsymbol{\nabla} \times\left(\frac{d}{d t}(\alpha \boldsymbol{u})\right)=-\frac{\nabla p \times \nabla \rho}{\rho^{2}}+\nabla \times \boldsymbol{E}_{g}-\boldsymbol{B}_{g}(\boldsymbol{\nabla} \cdot \boldsymbol{u})+\left(\boldsymbol{B}_{g} \cdot \boldsymbol{\nabla}\right) \boldsymbol{u}-(\boldsymbol{u} \cdot \boldsymbol{\nabla}) \boldsymbol{B}_{g}
$$


With the help of the continuity equation and Faraday's law:

$$
\boldsymbol{\nabla} \times\left(\frac{d}{d t}(\alpha \boldsymbol{u})\right)=-\frac{\nabla p \times \nabla \rho}{\rho^{2}}-\frac{d \boldsymbol{B}_{g}}{d t}+\frac{\boldsymbol{B}_{g}}{\rho} \frac{d \rho}{d t}+\left(\boldsymbol{B}_{g} \cdot \boldsymbol{\nabla}\right) \boldsymbol{u} .
$$

Substituting in the vorticity diffusion equation

$$
\frac{d}{d t}\left(\frac{\nabla \times(\alpha \boldsymbol{u})+\boldsymbol{B}_{g}}{\rho}\right)=\left(\frac{\boldsymbol{\nabla} \times(\alpha \boldsymbol{u})+\boldsymbol{B}_{g}}{\rho} \cdot \nabla\right) \boldsymbol{u}-\frac{\boldsymbol{\nabla} \times \boldsymbol{\nabla} \rho}{\rho^{3}} .
$$

The perfect fluid flow is barotropic (in which the pressure $p$ and the mass density $\rho$ are directly related), so that the last term in the right-hand side vanishes. Now, a canonical vorticity $\boldsymbol{\Omega}$ can be defined by

$$
\boldsymbol{\Omega}=\nabla \times \boldsymbol{P}
$$

where

$$
\boldsymbol{P}=m\left(\alpha \boldsymbol{u}+\boldsymbol{A}_{g}\right)
$$

is the canonical momentum of the fluid element defined in Subsection II A (the specific canonical momentum is given by the Clebsch representation (36)). The diffusion equation for the canonical vorticity becomes

$$
\frac{d}{d t}\left(\frac{\boldsymbol{\Omega}}{\rho}\right)=\left(\frac{\boldsymbol{\Omega}}{\rho} \cdot \nabla\right) \boldsymbol{u} .
$$

Introducing a change in the dependent variables such that [21]

$$
\frac{\Omega}{\rho}=C \cdot \nabla_{0} r
$$

the diffusion equation for $\Omega / \rho$ becomes

$$
\frac{d}{d t}\left(\boldsymbol{C} \cdot \nabla_{0} \boldsymbol{r}\right)=\left[\left(\boldsymbol{C} \cdot \nabla_{0} \boldsymbol{r}\right) \cdot \boldsymbol{\nabla}\right] \boldsymbol{u}
$$

Here $\boldsymbol{\nabla}_{0} \boldsymbol{r}=\partial \boldsymbol{r} / \partial \boldsymbol{r}_{0}=\overline{\overline{\boldsymbol{J}}}$ is the Jacobian dyadic of the transformation $\boldsymbol{r}=\boldsymbol{r}\left(\boldsymbol{r}_{0}, t\right)$ from the Lagrangian $\boldsymbol{r}_{0}$ to the Eulerian $\boldsymbol{r}$ coordinates $\left(|\overline{\bar{J}}| \neq 0\right.$ and $\left.\nabla_{0} \equiv \overline{\overline{\boldsymbol{J}}} \cdot \boldsymbol{\nabla}\right)$. The transformation $\boldsymbol{r}=\boldsymbol{r}\left(\boldsymbol{r}_{0}, t\right)$ specifies the trajectory of a fluid element. For fixed $t$, it determines the transformation of the element from the initial position $\boldsymbol{r}_{0}$ to the position $\boldsymbol{r}$ at time $t$ (Lagrangian map). Since

$$
\begin{aligned}
\frac{d}{d t}\left(\boldsymbol{C} \cdot \nabla_{0} \boldsymbol{r}\right) & =\frac{d \boldsymbol{C}}{d t} \cdot \nabla_{0} \boldsymbol{r}+\boldsymbol{C} \cdot \nabla_{0} \boldsymbol{u} \\
& =\frac{d \boldsymbol{C}}{d t} \cdot \nabla_{0} \boldsymbol{r}+\boldsymbol{C} \cdot \overline{\overline{\boldsymbol{J}}} \cdot \boldsymbol{\nabla} \boldsymbol{u} \\
& =\frac{d \boldsymbol{C}}{d t} \cdot \nabla_{0} \boldsymbol{r}+\left(\boldsymbol{C} \cdot \nabla_{0} \boldsymbol{r}\right) \cdot \boldsymbol{\nabla u}
\end{aligned}
$$

the diffusion equation for the canonical vorticity divided by the mass density reduces to

$$
\frac{d \boldsymbol{C}}{d t} \cdot \nabla_{0} \boldsymbol{r}=0 \Longrightarrow \frac{d \boldsymbol{C}}{d t}=0 \Longrightarrow \boldsymbol{C}=\boldsymbol{C}\left(\boldsymbol{r}_{0}\right)
$$

Thus

$$
\frac{\Omega}{\rho}=C\left(\boldsymbol{r}_{0}\right) \cdot \nabla_{0} \boldsymbol{r}
$$

Setting $t=0$

$$
\frac{\Omega}{\rho}=\frac{\Omega_{0}}{\rho_{0}} \cdot \nabla_{0} r
$$

This result was obtained, for an incompressible fluid in the non relativistic limit, and without the gravitomagnetic field, by Cauchy in 1815 [28, 29]. Cauchy demonstrated that a fluid element that is initially in irrotational motion remains in this condition throughout the flow. However, the hydrogravitomagnetic Cauchy invariant shows that the gravitomagnetic field may introduce flow vorticity in an otherwise irrotational motion. This has important implications in the rotation curve of galaxies [18] and in the mass accretion of astrophysical systems. 


\section{COVARIANT FORM OF THE GRAVITOELECTROMAGNETIC FLUID EQUATIONS}

In this section the gravitoelectromagnetic fluid equations of motion are written in covariant form according to the notation briefly presented in the next subsection.

\section{A. Preliminary remarks [30]}

The contravariant coordinates four-vector is

$$
x^{\mu}=(c t, \boldsymbol{r}),
$$

with the corresponding covariant form

$$
x_{\mu}=\eta_{\mu \nu} x^{\nu}=(-c t, \boldsymbol{r})
$$

where

$$
\eta_{\mu \nu}=\left(\begin{array}{cc}
-1 & \mathbf{0} \\
\mathbf{0} & \overline{\bar{I}}
\end{array}\right)=\eta^{\mu \nu}
$$

is the Minkowski (flat-space metric) tensor. Note that

$$
\eta^{\mu \rho} \eta_{\nu \rho}=\left(\begin{array}{cc}
1 & \mathbf{0} \\
\mathbf{0} & \overline{\overline{\boldsymbol{I}}}
\end{array}\right)=\delta_{\nu}^{\mu}
$$

is the Kronecker mixed tensor. Also,

$$
\eta^{\mu \nu} \eta_{\mu \nu}=4
$$

The covariant gradient four-vector is

$$
\partial_{\mu} \equiv \frac{\partial}{\partial x^{\mu}} \equiv\left(\frac{1}{c} \frac{\partial}{\partial t}, \nabla\right)
$$

with the corresponding contravariant form

$$
\partial^{\mu} \equiv \eta^{\mu \nu} \partial_{\nu} \equiv\left(-\frac{1}{c} \frac{\partial}{\partial t}, \nabla\right) .
$$

In general, the invariant scalar product of two four-vectors is

$$
a_{\mu} b^{\mu}=a_{0} b^{0}+\boldsymbol{a} \cdot \boldsymbol{b}=-a_{0} b_{0}+\boldsymbol{a} \cdot \boldsymbol{b} .
$$

In particular,

$$
x^{2}=x_{\mu} x^{\mu}=-c^{2} t^{2}+\boldsymbol{r}^{2} .
$$

The scalar product of $\partial_{\mu}$ with itself gives the d'Alembertian operator

$$
\square^{2} \equiv \partial_{\mu} \partial^{\mu} \equiv \eta^{\mu \nu} \frac{\partial}{\partial x^{\mu}} \frac{\partial}{\partial x^{\nu}} \equiv-\frac{1}{c^{2}} \frac{\partial^{2}}{\partial t^{2}}+\nabla^{2}
$$

which is also invariant. The proper time interval $d \tau$ is defined by

$$
c^{2} d \tau^{2}=-\eta_{\mu \nu} d x^{\mu} d x^{\nu}=c^{2} d t^{2}-d \boldsymbol{r}^{2}=c^{2}\left(1-\frac{\boldsymbol{u}^{2}}{c^{2}}\right) d t^{2},
$$

where

$$
\boldsymbol{u}=\frac{d \boldsymbol{r}}{d t}
$$


is the fluid velocity. The contravariant form of the fluid four-velocity is

$$
u^{\mu}=\frac{d x^{\mu}}{d \tau}=\gamma(c, \boldsymbol{u})
$$

where

$$
\gamma=\frac{d t}{d \tau}=\left(1-\frac{\boldsymbol{u}^{2}}{c^{2}}\right)^{-1 / 2}=\frac{1}{\sqrt{1-u^{2} / c^{2}}}=\frac{1}{\sqrt{1-\beta^{2}}}
$$

The scalar product gives

$$
u_{\mu} u^{\mu}=\gamma(-c, \boldsymbol{u}) \cdot \gamma(c, \boldsymbol{u})=\gamma^{2}\left(-c^{2}+u^{2}\right)=-c^{2}
$$

\section{B. Covariant formulation of the equations of motion}

The contravariant mass current density four-vector is defined by

$$
j^{\mu}=(\rho c, \boldsymbol{j}),
$$

so that the equation of continuity becomes

$$
\partial_{\mu} j^{\mu}=\left(\frac{1}{c} \frac{\partial}{\partial t}, \nabla\right) \cdot(\rho c, \boldsymbol{j})=\frac{\partial \rho}{\partial t}+\nabla \cdot \boldsymbol{j}=0 .
$$

The energy-momentum tensor of a perfect fluid can be defined as

$$
\begin{aligned}
& T_{f}^{\mu \nu}=p \eta^{\mu \nu}+(\stackrel{\circ}{U}+p) \frac{u^{\mu} u^{\nu}}{c^{2}} \\
& =p\left(\begin{array}{cc}
-1 & \mathbf{0} \\
\mathbf{0} & \overline{\overline{\boldsymbol{I}}}
\end{array}\right)+(\stackrel{\circ}{U}+p) \gamma^{2}\left(\begin{array}{cc}
1 & \boldsymbol{u} / c \\
\boldsymbol{u} / c & \boldsymbol{u} \boldsymbol{u} / c^{2}
\end{array}\right) \\
& =\left(\begin{array}{cc}
\gamma^{2}\left(\stackrel{\circ}{U}+p \beta^{2}\right) & (\stackrel{\circ}{U}+p) \gamma^{2} \boldsymbol{u} / c \\
(\stackrel{\circ}{U}+p) \gamma^{2} \boldsymbol{u} / c & p \overline{\overline{\boldsymbol{I}}}+(\stackrel{\circ}{U}+p) \gamma^{2} \boldsymbol{u u} / c^{2}
\end{array}\right),
\end{aligned}
$$

where $\stackrel{\circ}{U}=\stackrel{\circ}{n} m c^{2}+p /\left(\gamma_{A}-1\right)$ is the proper energy density, $p=\stackrel{\circ}{n} k_{B} \stackrel{\circ}{T}=n k_{B} T$ is the invariant pressure, and $\gamma_{A}$ is the "adiabatic" coefficient. This definition is in accordance with the equations of motion obtained by variational methods in Section II, as will be shown in the following. Indeed, in terms of the gravitoelectromagnetic field tensor $F^{\mu \nu}$ introduced in Subsection II A, the fluid energy-momentum equation can be written as

$$
\partial_{\nu} T_{f}^{\mu \nu}=j_{\nu} F^{\mu \nu}
$$

Thus

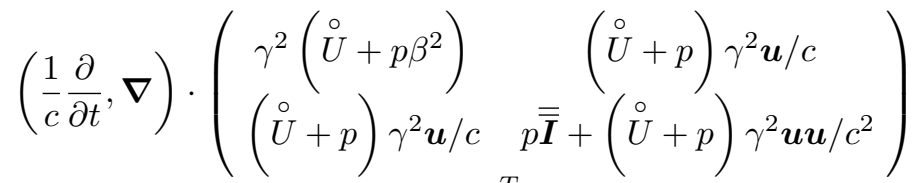

$$
\begin{aligned}
& =(-\rho c, \boldsymbol{j}) \cdot\left(\begin{array}{cc}
0 & \boldsymbol{E}_{g} / c \\
-\boldsymbol{E}_{g} / c & \overline{\overline{\boldsymbol{\epsilon}}} \cdot \boldsymbol{B}_{g}
\end{array}\right)^{T}=(-\rho c, \boldsymbol{j}) \cdot\left(\begin{array}{cc}
0 & -\boldsymbol{E}_{g} / c \\
\boldsymbol{E}_{g} / c & -\overline{\overline{\boldsymbol{\epsilon}}} \cdot \boldsymbol{B}_{g}
\end{array}\right) \text {. }
\end{aligned}
$$

The energy conservation equation is given by the temporal component of the previous equation

$$
\frac{1}{c} \frac{\partial}{\partial t}\left[\gamma^{2}\left(\stackrel{\circ}{U}+p \beta^{2}\right)\right]+\nabla \cdot\left[(\stackrel{\circ}{U}+p) \gamma^{2} \frac{\boldsymbol{u}}{c}\right]=\boldsymbol{j} \cdot \frac{\boldsymbol{E}_{g}}{c},
$$

and the momentum conservation equation is given by the spatial components

$$
\frac{1}{c} \frac{\partial}{\partial t}\left[(\stackrel{\circ}{U}+p) \gamma^{2} \frac{\boldsymbol{u}}{c}\right]+\nabla \cdot\left[(\stackrel{\circ}{U}+p) \gamma^{2} \frac{\boldsymbol{u} \boldsymbol{u}}{c^{2}}+p \overline{\overline{\boldsymbol{I}}}\right]=\rho \boldsymbol{E}_{g}+\boldsymbol{j} \times \boldsymbol{B}_{g} .
$$


Note that $-\boldsymbol{j} \cdot\left(\overline{\overline{\boldsymbol{\epsilon}}} \cdot \boldsymbol{B}_{g}\right)=\boldsymbol{j} \times \boldsymbol{B}_{g}$. With some rearrangement these equations can be written as

$$
\frac{\partial}{\partial t}\left[\gamma \rho c^{2}+\left(\frac{1}{\gamma_{A}-1}+\beta^{2}\right) \gamma^{2} p\right]+\nabla \cdot\left[\left(\gamma \rho c^{2}+\frac{\gamma_{A}}{\gamma_{A}-1} \gamma^{2} p\right) \boldsymbol{u}\right]=\boldsymbol{j} \cdot \boldsymbol{E}_{g}
$$

and

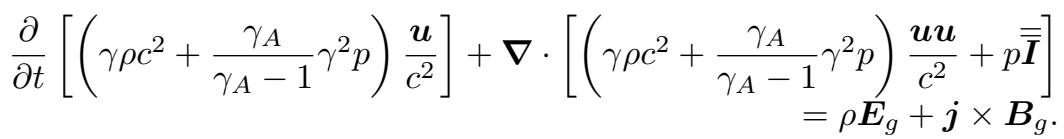

This shows that the above definition of the fluid energy-momentum tensor $T_{f}^{\mu \nu}$ gives the correct form of the energy and momentum density conservation equations (cf. equations (74) and (64)). The energy conservation equation can be obtained by scalar multiplication of the momentum conservation equation by $\boldsymbol{u}$ and application of the fluid continuity constraint, as demonstrated in Subsection II A.

The condition of entropy conservation is satisfied by the above covariant set of equations constrained by the second law of thermodynamics. This can be shown as follows. Multiplication of the energy-momentum conservation equation by the four-velocity yields

$$
\begin{aligned}
u_{\mu} \partial_{\nu} T_{f}^{\mu \nu} & =u_{\mu} j_{\nu} F^{\mu \nu}=\stackrel{\circ}{n} m u_{\mu} u_{\nu} F^{\mu \nu} \\
& =\stackrel{\circ}{n} m \gamma^{2}(-c, \boldsymbol{u}) \cdot\left(\begin{array}{cc}
0 & \boldsymbol{E}_{g} / c \\
-\boldsymbol{E}_{g} / c & \overline{\overline{\boldsymbol{\epsilon}}} \cdot \boldsymbol{B}_{g}
\end{array}\right) \cdot(-c, \boldsymbol{u}) \\
& =\stackrel{\circ}{n} m \gamma^{2}(-c, \boldsymbol{u}) \cdot\left(\begin{array}{c}
-\boldsymbol{u} \cdot \boldsymbol{E}_{g} / c \\
-\boldsymbol{E}_{g}-\boldsymbol{u} \times \boldsymbol{B}_{g}
\end{array}\right)=0 .
\end{aligned}
$$

Hence, replacing $T_{f}^{\mu \nu}$ by its covariant form,

$$
\begin{aligned}
u_{\mu} \partial_{\nu} T_{f}^{\mu \nu} & =u_{\mu} \partial_{\nu}\left[p \eta^{\mu \nu}+(\stackrel{\circ}{U}+p) \frac{u^{\mu} u^{\nu}}{c^{2}}\right] \\
& =u_{\mu} \partial^{\mu} p-\partial_{\nu}\left[(\stackrel{\circ}{U}+p) u^{\nu}\right]+(\stackrel{\circ}{U}+p) \frac{u^{\nu} u_{\mu}}{c^{2} \partial_{\nu} u^{\mu}} \\
& =u_{\mu} \partial^{\mu} p-\partial_{\nu}\left[(\stackrel{\circ}{U}+p) u^{\nu}\right]+\frac{1}{2}(\stackrel{\circ}{U}+p) u^{\nu} \underbrace{\partial_{\nu}\left(\frac{u_{\mu} u^{\mu}}{c^{2}}\right)}_{=0}=0
\end{aligned}
$$

Using the equation of continuity

$$
\partial_{\mu}\left(\stackrel{\circ}{n} u^{\mu}\right)=0
$$

the previous equation becomes

$$
\stackrel{\circ}{n} u^{\mu}\left[p \partial_{\mu}(1 / \stackrel{\circ}{n})+\partial_{\mu}(\stackrel{\circ}{U} / \stackrel{\circ}{n})\right]=0 .
$$

The second law of thermodynamics (23)

$$
\stackrel{\circ}{T} d s=d(\stackrel{\circ}{U} / \stackrel{\circ}{n})+p d(1 / \stackrel{\circ}{n})
$$

gives

$$
\stackrel{\circ}{n} \stackrel{\circ}{T} u^{\mu} \partial_{\mu} s=p u^{\mu} \partial_{\mu} s=0,
$$

where $s$ is the invariant specific entropy of the perfect fluid. 


\section{Covariant formulation of Maxwell's equations}

In covariant form the Maxwell source equations are written as

$$
\partial_{\nu} F^{\mu \nu}=-\frac{4 \pi G}{c^{2}} j^{\mu}
$$

In components form

$$
\left(\frac{1}{c} \frac{\partial}{\partial t}, \nabla\right) \cdot\left(\begin{array}{cc}
0 & \boldsymbol{E}_{g} / c \\
-\boldsymbol{E}_{g} / c & \overline{\overline{\boldsymbol{\epsilon}}} \cdot \boldsymbol{B}_{g}
\end{array}\right)^{T}=-\frac{4 \pi G}{c^{2}}(\rho c, \boldsymbol{j}),
$$

and, noting that $\boldsymbol{\nabla} \cdot\left(\overline{\overline{\boldsymbol{\epsilon}}} \cdot \boldsymbol{B}_{g}\right)^{T}=\boldsymbol{\nabla} \cdot\left(\overline{\overline{\boldsymbol{\epsilon}}}^{T} \cdot \boldsymbol{B}_{g}\right)=-\boldsymbol{\nabla} \cdot\left(\overline{\overline{\boldsymbol{\epsilon}}} \cdot \boldsymbol{B}_{g}\right)=-\boldsymbol{\nabla} \times \boldsymbol{B}_{g}$, it follows that

$$
\begin{aligned}
\nabla \cdot \boldsymbol{E}_{g} & =-4 \pi G \rho \\
\boldsymbol{\nabla} \times \boldsymbol{B}_{g} & =-\frac{4 \pi G}{c^{2}} \boldsymbol{j}+\frac{1}{c^{2}} \frac{\partial \boldsymbol{E}_{g}}{\partial t} .
\end{aligned}
$$

The anti-symmetric gravitoelectromagnetic field tensor can be derived from the four-vector potential $A_{g}^{\mu}=$ $\left(\phi_{g} / c, \boldsymbol{A}_{g}\right)$ as follows

$$
F^{\mu \nu}=\partial^{\mu} A_{g}^{\nu}-\partial^{\nu} A_{g}^{\mu}
$$

that is,

$$
\begin{aligned}
\left(\begin{array}{cc}
0 & \boldsymbol{E}_{g} / c \\
-\boldsymbol{E}_{g} / c & \overline{\overline{\boldsymbol{\epsilon}}} \cdot \boldsymbol{B}_{g}
\end{array}\right) & =\left(\begin{array}{cc}
-\frac{1}{c} \frac{\partial}{\partial t} \\
\boldsymbol{\nabla}
\end{array}\right)\left(\begin{array}{cc}
\frac{\phi_{g}}{c} & \boldsymbol{A}_{g}
\end{array}\right)-\left[\left(\begin{array}{cc}
-\frac{1}{c} \frac{\partial}{\partial t} \\
\nabla
\end{array}\right)\left(\begin{array}{cc}
\frac{\phi_{g}}{c} & \boldsymbol{A}_{g}
\end{array}\right)\right]^{T} \\
& =\left(\begin{array}{cc}
0 & -\frac{1}{c} \frac{\partial \boldsymbol{A}_{g}}{\partial t}-\frac{1}{c} \boldsymbol{\nabla} \phi_{g} \\
\frac{1}{c} \boldsymbol{\nabla} \phi_{g}+\frac{1}{c} \frac{\partial \boldsymbol{A}_{g}}{\partial t} & \boldsymbol{\nabla} \boldsymbol{A}_{g}-\left(\boldsymbol{\nabla} \boldsymbol{A}_{g}\right)^{T}
\end{array}\right) .
\end{aligned}
$$

Hence

$$
\begin{aligned}
& \boldsymbol{E}_{g}=-\boldsymbol{\nabla} \phi_{g}-\frac{\partial \boldsymbol{A}_{g}}{\partial t} \\
& \boldsymbol{B}_{g}=\boldsymbol{\nabla} \times \boldsymbol{A}_{g}
\end{aligned}
$$

The relation $F^{\mu \nu}=\partial^{\mu} A_{g}^{\nu}-\partial^{\nu} A_{g}^{\mu}$ is a consequence of gauge invariance of the second kind. The quantities $A_{g}^{\mu}(x)$ and $A_{g}^{\mu}(x)-\partial^{\mu} f(x)$ are physically indistinguishable, so that $A_{g}^{\mu}$ can be required to satisfy Lorenz's condition

$$
\partial_{\mu} A_{g}^{\mu}=0 \rightarrow \frac{1}{c^{2}} \frac{\partial \phi_{g}}{\partial t}+\nabla \cdot \boldsymbol{A}_{g}=0
$$

The inhomogeneous field equations can be written in terms of the four-potential as

$$
\square^{2} A_{g}^{\mu}=\frac{4 \pi G}{c^{2}} j^{\mu}\left\{\begin{aligned}
\square^{2} \phi_{g} & =4 \pi G \rho \\
\square^{2} \boldsymbol{A}_{g} & =\frac{4 \pi G}{c^{2}} \boldsymbol{j}
\end{aligned}\right.
$$

Summary: The dynamics of a fully relativistic fluid in the flat-space gravitoelectromagnetic field is described by the covariant set of equations

$$
\left\{\begin{aligned}
\partial_{\nu} T_{f}^{\mu \nu} & =j_{\nu} F^{\mu \nu} & & \text { energy-momentum conservation } \\
\square^{2} A_{g}^{\mu} & =\frac{4 \pi G}{c^{2}} j^{\mu} & & \text { Maxwell's source equations } \\
p u^{\mu} \partial_{\mu} s & =0 & & \text { entropy conservation }
\end{aligned}\right.
$$

with

$$
F^{\mu \nu}=\partial^{\mu} A_{g}^{\nu}-\partial^{\nu} A_{g}^{\mu} \text { and } \partial_{\mu} A_{g}^{\mu}=0
$$


This set of covariant equations gives a total of 9 equations in the 5 fluid $(\rho, \boldsymbol{u}, p)$ and 4 field $\left(\phi_{g}, \boldsymbol{A}_{g}\right)$ variables. Note that the continuity condition is automatically satisfied by Maxwell's source equations. Note also that the energy-momentum equation constrained by the second law of thermodynamics satisfies the condition of isentropic flow. Taking into account the second law only one equation of state, the perfect gas law in the present case, is needed to close the system of fluid-field equations.

\section{Lorentz transformation of the gravitoelectromagnetic field}

A restricted or proper Lorentz transformation by the four-velocity $v^{\mu}=\gamma(c, \boldsymbol{v})$ is described by the matrix (pure boost)

$$
\Lambda_{\nu}^{\mu}=\left(\begin{array}{cc}
\gamma & \gamma \frac{\boldsymbol{v}}{c} \\
\gamma \frac{\boldsymbol{v}}{c} & \overline{\overline{\boldsymbol{I}}}+\frac{\gamma-1}{\beta^{2}} \frac{\boldsymbol{v} \boldsymbol{v}}{c^{2}}
\end{array}\right) .
$$

The transformation of the gravitoelectromagnetic field $F^{\mu \nu}$ is given by

$$
\begin{aligned}
& F^{\mu \nu}=\Lambda_{\rho}{ }^{\mu} \Lambda_{\sigma}{ }^{\nu} F^{\rho \sigma}=\left[\Lambda^{T}(\boldsymbol{v}) F \Lambda(\boldsymbol{v})\right]^{\mu \nu} \\
& =\left(\begin{array}{cc}
\gamma & \gamma \frac{\boldsymbol{v}}{c} \\
\gamma \frac{\boldsymbol{v}}{c} & \overline{\overline{\boldsymbol{I}}}+\frac{\gamma-1}{\beta^{2}} \frac{\boldsymbol{v} \boldsymbol{v}}{c^{2}}
\end{array}\right) \cdot\left(\begin{array}{cc}
0 & \frac{\boldsymbol{E}_{g}}{c} \\
-\frac{\boldsymbol{E}_{g}}{c} & \overline{\overline{\boldsymbol{\epsilon}}} \cdot \boldsymbol{B}_{g}
\end{array}\right) \cdot\left(\begin{array}{cc}
\gamma & \gamma \frac{\boldsymbol{v}}{c} \\
\gamma \frac{\boldsymbol{v}}{c} & \overline{\overline{\boldsymbol{I}}}+\frac{\gamma-1}{\beta^{2}} \frac{\boldsymbol{v} \boldsymbol{v}}{c^{2}}
\end{array}\right) .
\end{aligned}
$$

Hence

$$
\begin{aligned}
& F^{\prime \mu \nu}=\left(\begin{array}{cc}
\gamma & \gamma \frac{\boldsymbol{v}}{c} \\
\gamma \frac{\boldsymbol{v}}{c} & \overline{\overline{\boldsymbol{I}}}+\frac{\gamma-1}{\beta^{2}} \frac{\boldsymbol{v} \boldsymbol{v}}{c^{2}}
\end{array}\right) \\
& \cdot\left(\begin{array}{cc}
\gamma \frac{\boldsymbol{v} \cdot \boldsymbol{E}_{g}}{c^{2}} & \frac{\boldsymbol{E}_{g}}{c}+\frac{\gamma-1}{\beta^{2}} \frac{\boldsymbol{v} \boldsymbol{v} \cdot \boldsymbol{E}_{g}}{c^{3}} \\
-\gamma \frac{\boldsymbol{E}_{g}}{c}+\gamma \frac{\boldsymbol{v} \times \boldsymbol{B}_{g}}{c} & -\gamma \frac{\boldsymbol{E}_{g} \boldsymbol{v}}{c^{2}}+\overline{\overline{\boldsymbol{\epsilon}}} \cdot \boldsymbol{B}_{g}+\frac{\gamma-1}{\beta^{2}} \frac{\left(\boldsymbol{v} \times \boldsymbol{B}_{g}\right) \boldsymbol{v}}{c^{2}}
\end{array}\right)
\end{aligned}
$$

and

$$
\begin{gathered}
F^{\prime \mu \nu}=\left(\begin{array}{c}
\boldsymbol{E}_{g} \\
-\gamma \frac{\gamma^{2}}{c}+\frac{\boldsymbol{v} \boldsymbol{v} \cdot \boldsymbol{E}_{g}}{\gamma+1}+\gamma \frac{\boldsymbol{v} \times \boldsymbol{B}_{g}}{c} \\
c^{3} \\
\gamma \frac{\boldsymbol{E}_{g}}{c}-\frac{\gamma^{2}}{\gamma+1} \frac{\boldsymbol{v} \cdot \boldsymbol{E}_{g}}{c^{3}}-\gamma \frac{\boldsymbol{v} \times \boldsymbol{B}_{g}}{c} \\
-\gamma \frac{\boldsymbol{E}_{g} \boldsymbol{v}-\boldsymbol{v} \boldsymbol{E}_{g}}{c^{2}}+\overline{\overline{\boldsymbol{\epsilon}}} \cdot \boldsymbol{B}_{g}-\frac{\gamma-1}{\beta^{2}} \frac{\boldsymbol{v}\left(\boldsymbol{v} \times \boldsymbol{B}_{g}\right)-\left(\boldsymbol{v} \times \boldsymbol{B}_{g}\right) \boldsymbol{v}}{c^{2}}
\end{array}\right),
\end{gathered}
$$

so that the fields in the primed system, moving with velocity $\boldsymbol{v}$ relative to the unprimed frame, can be written as

$$
\left\{\begin{aligned}
\boldsymbol{E}_{g}^{\prime} & =\gamma\left(\overline{\overline{\boldsymbol{I}}}-\frac{\gamma}{\gamma+1} \frac{\boldsymbol{v} \boldsymbol{v}}{c^{2}}\right) \cdot \boldsymbol{E}_{g}-\gamma \boldsymbol{v} \times \boldsymbol{B}_{g} \\
\overline{\overline{\boldsymbol{\epsilon}}} \cdot \boldsymbol{B}_{g}^{\prime} & =\overline{\overline{\boldsymbol{\epsilon}}} \cdot \boldsymbol{B}_{g}-\frac{\gamma-1}{\beta^{2}} \frac{\boldsymbol{v}\left(\boldsymbol{v} \times \boldsymbol{B}_{g}\right)-\left(\boldsymbol{v} \times \boldsymbol{B}_{g}\right) \boldsymbol{v}}{c^{2}}-\gamma \frac{\boldsymbol{E}_{g} \boldsymbol{v}-\boldsymbol{v} \boldsymbol{E}_{g}}{c^{2}}
\end{aligned}\right.
$$

Using the identity

$$
\frac{\boldsymbol{v}}{c} \times\left(\frac{\boldsymbol{v}}{c} \times \boldsymbol{E}_{g}\right)=\left(\frac{\boldsymbol{v} \boldsymbol{v}}{c^{2}}-\frac{v^{2}}{c^{2}} \overline{\overline{\boldsymbol{I}}}\right) \cdot \boldsymbol{E}_{g},
$$

the transformation of the GE field becomes

$$
\boldsymbol{E}_{g}^{\prime}=\boldsymbol{E}_{g}-\frac{\gamma^{2}}{\gamma+1} \frac{\boldsymbol{v} \times\left(\boldsymbol{v} \times \boldsymbol{E}_{g}\right)}{c^{2}}-\gamma \boldsymbol{v} \times \boldsymbol{B}_{g} .
$$


Moreover, using the identities

$$
\begin{aligned}
-\frac{\gamma-1}{\beta^{2}}\left(\frac{\boldsymbol{v}\left(\boldsymbol{v} \times \boldsymbol{B}_{g}\right)-\left(\boldsymbol{v} \times \boldsymbol{B}_{g}\right) \boldsymbol{v}}{c^{2}}\right) & =-\frac{\gamma^{2}}{\gamma+1} \overline{\overline{\overline{\boldsymbol{\epsilon}}}} \cdot\left(\frac{\boldsymbol{v} \times\left(\boldsymbol{v} \times \boldsymbol{B}_{g}\right)}{c^{2}}\right), \\
-\gamma\left(\frac{\boldsymbol{E}_{g} \boldsymbol{v}-\boldsymbol{v} \boldsymbol{E}_{g}}{c^{2}}\right) & =\gamma \overline{\overline{\boldsymbol{\epsilon}}} \cdot\left(\frac{\boldsymbol{v} \times \boldsymbol{E}_{g}}{c^{2}}\right),
\end{aligned}
$$

the transformation of the GM field becomes

$$
\boldsymbol{B}_{g}^{\prime}=\boldsymbol{B}_{g}-\frac{\gamma^{2}}{\gamma+1} \frac{\boldsymbol{v} \times\left(\boldsymbol{v} \times \boldsymbol{B}_{g}\right)}{c^{2}}+\gamma \frac{\boldsymbol{v} \times \boldsymbol{E}_{g}}{c^{2}} .
$$

In components parallel and perpendicular to $\boldsymbol{v}$ :

$$
\left\{\begin{aligned}
E_{g \|}^{\prime} & =E_{g \|} \\
\boldsymbol{E}_{g \perp}^{\prime} & =\gamma\left(\boldsymbol{E}_{g \perp}-\boldsymbol{v} \times \boldsymbol{B}_{g \perp}\right) \\
B_{g \|}^{\prime} & =B_{g \|} \\
\boldsymbol{B}_{g \perp}^{\prime} & =\gamma\left(\boldsymbol{B}_{g \perp}+\boldsymbol{v} \times \boldsymbol{E}_{g \perp} / c^{2}\right)
\end{aligned}\right.
$$

Thus, the transformation of the gravitoelectromagnetic fields is identical to the transformation of the fields in electromagnetic theory [31, 32]. Note that the Lorentz transformation $\Lambda(\boldsymbol{v})$ transports a fluid element from rest to velocity $\boldsymbol{v}$. The inverse transformation $\Lambda(-\boldsymbol{v})$ transports the element to the rest frame.

\section{CONSERVATION EQUATIONS}

The conservation equations can be written in a compact form introducing the momentum, stress and energy densities for the fluid:

$$
\begin{aligned}
& \boldsymbol{G}_{f}=\left(\gamma \rho+\frac{\gamma_{A}}{\gamma_{A}-1} \frac{\gamma^{2} p}{c^{2}}\right) \boldsymbol{u} \quad \text { fluid momentum density } \\
& \overline{\overline{\boldsymbol{T}}}_{f}=\gamma \rho \boldsymbol{u} \boldsymbol{u}+\left(\overline{\overline{\boldsymbol{I}}}+\frac{\gamma_{A}}{\gamma_{A}-1} \gamma^{2} \frac{\boldsymbol{u u}}{c^{2}}\right) p \quad \text { fluid stress tensor } \\
& U_{f}=\gamma \rho c^{2}+\left(\frac{1}{\gamma_{A}-1}+\beta^{2}\right) \gamma^{2} p \quad \text { fluid energy density }
\end{aligned}
$$

Hence

$$
\begin{aligned}
\frac{\partial \boldsymbol{G}_{f}}{\partial t}+\boldsymbol{\nabla} \cdot \overline{\bar{T}}_{f} & =\rho \boldsymbol{E}_{g}+\boldsymbol{j} \times \boldsymbol{B}_{g} & & \text { momentum conservation equation } \\
\frac{\partial U_{f}}{\partial t}+c^{2} \boldsymbol{\nabla} \cdot \boldsymbol{G}_{f} & =\boldsymbol{j} \cdot \boldsymbol{E}_{g} & & \text { energy conservation equation }
\end{aligned}
$$

Note the following relations:

$$
\left\{\begin{aligned}
\overline{\overline{\boldsymbol{T}}}_{f}: \overline{\overline{\boldsymbol{I}}} & =\gamma \rho u^{2}+\left(3+\frac{\gamma_{A}}{\gamma_{A}-1} \gamma^{2} \beta^{2}\right) p \\
\overline{\overline{\boldsymbol{T}}}_{f}: \frac{\boldsymbol{u u}}{u^{2}} & =\gamma \rho u^{2}+\left(1+\frac{\gamma_{A}}{\gamma_{A}-1} \gamma^{2} \beta^{2}\right) p=\beta^{2} U_{f}+\left(1+\beta^{2}\right) p \\
2 p & =\overline{\overline{\boldsymbol{T}}}_{f}:\left(\overline{\overline{\boldsymbol{I}}}-\frac{\boldsymbol{u} \boldsymbol{u}}{u^{2}}\right) \\
\beta^{2} U_{f} & =\overline{\overline{\boldsymbol{T}}}_{f}: \frac{\boldsymbol{u} \boldsymbol{u}}{u^{2}}-\left(\frac{1+\beta^{2}}{2}\right) \overline{\overline{\boldsymbol{T}}}_{f}:\left(\overline{\overline{\boldsymbol{I}}}-\frac{\boldsymbol{u} \boldsymbol{u}}{u^{2}}\right)
\end{aligned}\right.
$$

Using the gravitoelectromagnetic laws of Gauss, Ampère and Faraday, and standard procedures [31-33], the mass and current mass sources can be eliminated so that the momentum conservation equation can be written as

$$
\begin{aligned}
\frac{\partial \boldsymbol{G}_{f}}{\partial t}+\nabla \cdot \overline{\overline{\boldsymbol{T}}}_{f}= & \frac{1}{4 \pi G} \frac{\partial}{\partial t}\left(\boldsymbol{E}_{g} \times \boldsymbol{B}_{g}\right) \\
& +\frac{1}{4 \pi G} \boldsymbol{\nabla} \cdot\left(\frac{E_{g}^{2}}{2} \overline{\overline{\boldsymbol{I}}}-\boldsymbol{E}_{g} \boldsymbol{E}_{g}\right)+\frac{c^{2}}{4 \pi G} \boldsymbol{\nabla} \cdot\left(\frac{B_{g}^{2}}{2} \overline{\overline{\boldsymbol{I}}}-\boldsymbol{B}_{g} \boldsymbol{B}_{g}\right) .
\end{aligned}
$$


Similarly, the energy conservation equation becomes

$$
\frac{\partial U_{f}}{\partial t}+c^{2} \boldsymbol{\nabla} \cdot \boldsymbol{G}_{f}=\frac{1}{4 \pi G} \frac{\partial}{\partial t}\left(\frac{E_{g}^{2}+c^{2} B_{g}^{2}}{2}\right)+\frac{c^{2}}{4 \pi G} \boldsymbol{\nabla} \cdot\left(\boldsymbol{E}_{g} \times \boldsymbol{B}_{g}\right) .
$$

Therefore, the momentum and energy conservation equations can be written as

$$
\begin{aligned}
\frac{\partial}{\partial t}\left(\boldsymbol{G}_{f}+\boldsymbol{G}_{g}\right) & =-\boldsymbol{\nabla} \cdot\left(\overline{\overline{\boldsymbol{T}}}_{f}+\overline{\overline{\boldsymbol{T}}}_{g}^{(e)}+\overline{\overline{\boldsymbol{T}}}_{g}^{(m)}\right), \\
\frac{\partial}{\partial t}\left(U_{f}+U_{g}\right) & =-c^{2} \boldsymbol{\nabla} \cdot\left(\boldsymbol{G}_{f}+\boldsymbol{G}_{g}\right) .
\end{aligned}
$$

where

$$
\begin{aligned}
\overline{\overline{\boldsymbol{T}}}_{g}^{(e)}=-\frac{1}{4 \pi G}\left(\frac{E_{g}^{2}}{2} \overline{\overline{\boldsymbol{I}}}-\boldsymbol{E}_{g} \boldsymbol{E}_{g}\right) & & \text { GE field stress tensor } \\
\overline{\overline{\boldsymbol{T}}}_{g}^{(m)}=-\frac{c^{2}}{4 \pi G}\left(\frac{B_{g}^{2}}{2} \overline{\overline{\boldsymbol{I}}}-\boldsymbol{B}_{g} \boldsymbol{B}_{g}\right) & & \text { GM field stress tensor } \\
\boldsymbol{G}_{g}=-\frac{1}{4 \pi G}\left(\boldsymbol{E}_{g} \times \boldsymbol{B}_{g}\right) & & \text { GEM field momentum density } \\
U_{g}=-\frac{E_{g}^{2}+c^{2} B_{g}^{2}}{8 \pi G}=\left(\overline{\overline{\boldsymbol{T}}}_{g}^{(e)}+\overline{\overline{\boldsymbol{T}}}_{g}^{(m)}\right): \overline{\overline{\boldsymbol{I}}} & & \text { GEM field energy density }
\end{aligned}
$$

Here, the free-field stress tensors $\overline{\overline{\boldsymbol{T}}}_{g}^{(e)}$ and $\overline{\overline{\boldsymbol{T}}}_{g}^{(m)}$ are defined in analogy to the Maxwell stress tensors in electromagnetism. The total gravitoelectromagnetic stress tensor $\overline{\overline{\boldsymbol{T}}}_{g}=\overline{\overline{\boldsymbol{T}}}_{g}^{(e)}+\overline{\overline{\boldsymbol{T}}}_{g}^{(m)}$ is given by the sum of the GE and GM stress tensors.

The total energy-momentum tensor $T^{\mu \nu}=T_{f}^{\mu \nu}+T_{g}^{\mu \nu}$ is given by the sum of the fluid $T_{f}^{\mu \nu}$ and the gravitoelectromagnetic $T_{g}^{\mu \nu}$ tensors:

$$
T_{f}^{\mu \nu}=\left(\begin{array}{cc}
U_{f} & c \boldsymbol{G}_{f} \\
c \boldsymbol{G}_{f} & \overline{\bar{T}}_{f}
\end{array}\right) \text { and } T_{g}^{\mu \nu}=\left(\begin{array}{cc}
U_{g} & c \boldsymbol{G}_{g} \\
c \boldsymbol{G}_{g} & \overline{\overline{\boldsymbol{T}}}_{g}
\end{array}\right) .
$$

Hence, the total momentum and energy conservation equations can be written in covariant form as follows

$$
\partial_{\nu} T^{\mu \nu}=0
$$

This equation describes the exchange of energy between matter and the gravitoelectromagnetic field in flat-space.

\section{A. Integral form of the conservation theorems}

The system of equations of motion for a flowing mass distribution in the gravitoelectromagnetic field is given in conservation form by

$$
\begin{aligned}
\partial \rho / \partial t & =-\boldsymbol{\nabla} \cdot \boldsymbol{j} & & \text { mass density conservation } \\
\partial \boldsymbol{G} / \partial t & =-\boldsymbol{\nabla} \cdot \overline{\overline{\boldsymbol{T}}} & & \text { momentum density conservation } \\
\partial U / \partial t & =-c^{2} \boldsymbol{\nabla} \cdot \boldsymbol{G} & & \text { energy density conservation }
\end{aligned}
$$

where

$$
\boldsymbol{G}=\boldsymbol{G}_{f}+\boldsymbol{G}_{g}, \overline{\overline{\boldsymbol{T}}}=\overline{\overline{\boldsymbol{T}}}_{f}+\overline{\overline{\boldsymbol{T}}}_{g} \text { and } U=U_{f}+U_{g}
$$

denote the total fluid and gravitoelectromagnetic field momentum density, stress dyadic and energy density, respectively. Integration over a volume $V$ gives the integral form of the conservation equations:

$$
\begin{aligned}
\int_{V} \dot{\rho} d^{3} r & =-\oint_{S} \boldsymbol{j} \cdot d^{2} \boldsymbol{r} & & \text { mass conservation } \\
\int_{V} \dot{\boldsymbol{G}} d^{3} r & =-\oint_{S} \overline{\overline{\boldsymbol{T}}} \cdot d^{2} \boldsymbol{r} & & \text { momentum conservation } \\
\int_{V} \dot{U} d^{3} r & =-c^{2} \oint_{S} \boldsymbol{G} \cdot d^{2} \boldsymbol{r} & & \text { energy conservation }
\end{aligned}
$$


Now, the laws of Ampère and Faraday give

$$
\begin{aligned}
& \boldsymbol{E}_{g} \cdot \nabla \times \boldsymbol{B}_{g}=-\frac{4 \pi G}{c^{2}} \boldsymbol{j} \cdot \boldsymbol{E}_{g}+\frac{1}{c^{2}} \boldsymbol{E}_{g} \cdot \dot{\boldsymbol{E}}_{g}, \\
& \boldsymbol{B}_{g} \cdot \boldsymbol{\nabla} \times \boldsymbol{E}_{g}=-\boldsymbol{B}_{g} \cdot \dot{\boldsymbol{B}}_{g} .
\end{aligned}
$$

The subtraction of these two equations gives

$$
\boldsymbol{\nabla} \cdot\left(-\frac{c^{2}}{4 \pi G} \boldsymbol{E}_{g} \times \boldsymbol{B}_{g}\right)=-\boldsymbol{j} \cdot \boldsymbol{E}_{g}+\frac{\dot{E}_{g}^{2}+c^{2} \dot{B}_{g}^{2}}{8 \pi G} .
$$

Thus

$$
\boldsymbol{\nabla} \cdot \boldsymbol{S}_{g}=-\boldsymbol{j} \cdot \boldsymbol{E}_{g}-\dot{U}_{g}
$$

where

$$
\boldsymbol{S}_{g}=-\frac{c^{2}}{4 \pi G} \boldsymbol{E}_{g} \times \boldsymbol{B}_{g}=c^{2} \boldsymbol{G}_{g}
$$

is the vector of Poynting. Integration over $V$ yields the theorem of Poynting

$$
\int_{V}\left(\dot{U}_{g}+\boldsymbol{j} \cdot \boldsymbol{E}_{g}\right) d^{3} r=-\oint_{S} \boldsymbol{S}_{g} \cdot d^{2} \boldsymbol{r}
$$

Multiplying the momentum density conservation equation by $\boldsymbol{r} \times$ gives

$$
\boldsymbol{r} \times \dot{\boldsymbol{G}}=-\boldsymbol{r} \times(\nabla \cdot \overline{\bar{T}}) .
$$

The angular momentum density is defined by $\boldsymbol{L}=\boldsymbol{r} \times \boldsymbol{G}$, so that

$$
\dot{L}=-\nabla \cdot\left(r \times \overline{\bar{T}}^{T}\right)+\overline{\bar{T}}_{\times}
$$

The superscript $T$ denotes the transposed dyadic and the subscript $\times$ the vector of a dyadic. The stress dyadic $\overline{\overline{\boldsymbol{T}}}$ is symmetric $\left(\overline{\overline{\boldsymbol{T}}}^{T}=\overline{\overline{\boldsymbol{T}}}, \overline{\overline{\boldsymbol{T}}}_{\times}=0\right)$, hence

$$
\dot{L}=-\nabla \cdot(r \times \overline{\bar{T}})=-\nabla \cdot \overline{\bar{M}}
$$

The flux of angular momentum is defined by $\overline{\overline{\boldsymbol{M}}}=\boldsymbol{r} \times \overline{\overline{\boldsymbol{T}}}$, and the last equation indicates that, due to both the conservation of momentum density and the symmetry of $\overline{\overline{\boldsymbol{T}}}$, the angular momentum density is also conserved. Integration over $V$ gives the conservation of angular momentum in integral form

$$
\int \dot{\boldsymbol{L}} d^{3} r=-\oint \overline{\overline{\boldsymbol{M}}} \cdot d^{2} \boldsymbol{r}
$$

Finally, consider the quantity

$$
\begin{aligned}
\nabla \cdot\left[\boldsymbol{r} \cdot\left(\overline{\overline{\boldsymbol{T}}}_{f}+\overline{\overline{\boldsymbol{T}}}_{g}\right)\right] & =(\boldsymbol{\nabla r}):\left(\overline{\overline{\boldsymbol{T}}}_{f}+\overline{\overline{\boldsymbol{T}}}_{g}\right)+\boldsymbol{r} \cdot \boldsymbol{\nabla} \cdot\left(\overline{\overline{\boldsymbol{T}}}_{f}+\overline{\overline{\boldsymbol{T}}}_{g}\right)^{T} \\
& =\overline{\overline{\boldsymbol{I}}}:\left(\overline{\overline{\boldsymbol{T}}}_{f}+\overline{\overline{\boldsymbol{T}}}_{g}\right)+\boldsymbol{r} \cdot \boldsymbol{\nabla} \cdot\left(\overline{\overline{\boldsymbol{T}}}_{f}+\overline{\overline{\boldsymbol{T}}}_{g}\right)
\end{aligned}
$$

that is

$$
\nabla \cdot\left[\boldsymbol{r} \cdot\left(\overline{\overline{\boldsymbol{T}}}_{f}+\overline{\overline{\boldsymbol{T}}}_{g}\right)\right]=\overline{\overline{\boldsymbol{I}}}:\left(\overline{\overline{\boldsymbol{T}}}_{f}+\overline{\overline{\boldsymbol{T}}}_{g}\right)-\boldsymbol{r} \cdot\left(\dot{\boldsymbol{G}}_{f}+\dot{\boldsymbol{G}}_{g}\right)
$$

Integration over $V$ yields

$$
\oint_{S}\left[\boldsymbol{r} \cdot\left(\overline{\overline{\boldsymbol{T}}}_{f}+\overline{\overline{\boldsymbol{T}}}_{g}\right)\right] \cdot d^{2} \boldsymbol{r}=\int_{V} \overline{\overline{\boldsymbol{I}}}:\left(\overline{\overline{\boldsymbol{T}}}_{f}+\overline{\overline{\boldsymbol{T}}}_{g}\right) d^{3} r-\int_{V} \boldsymbol{r} \cdot\left(\dot{\boldsymbol{G}}_{f}+\dot{\boldsymbol{G}}_{g}\right) d^{3} r .
$$


Assuming that all quantities are bounded, one takes the time average over a long period of time according to the definition

$$
\langle\dot{f}\rangle=\frac{1}{t} \int_{0}^{t} \dot{f} d t=\frac{f(t)-f(0)}{t} \underset{t \rightarrow \infty}{\rightarrow} 0 .
$$

Thus

$$
\int_{V}\left\langle\overline{\overline{\boldsymbol{I}}}:\left(\overline{\overline{\boldsymbol{T}}}_{f}+\overline{\overline{\boldsymbol{T}}}_{g}\right)\right\rangle d^{3} r=\oint_{S}\left\langle\boldsymbol{r} \cdot\left(\overline{\overline{\boldsymbol{T}}}_{f}+\overline{\overline{\boldsymbol{T}}}_{g}\right)\right\rangle \cdot d^{2} \boldsymbol{r} .
$$

This is a general form of the virial theorem in equilibrium [34]. Substituting the expressions for the stress dyadics:

$$
\begin{aligned}
& \int_{V}\left\langle\gamma \rho u^{2}+\left(3+\frac{\gamma_{A}}{\gamma_{A}-1} \gamma^{2} \beta^{2}\right) p+U_{g}\right\rangle d^{3} r \\
& =\oint_{S}\left\langle\boldsymbol{r} \cdot\left[\gamma \rho \boldsymbol{u u}+\left(\overline{\overline{\boldsymbol{I}}}+\frac{\gamma_{A}}{\gamma_{A}-1} \gamma^{2} \frac{\boldsymbol{u} \boldsymbol{u}}{c^{2}}\right) p\right]\right\rangle \cdot d^{2} \boldsymbol{r} \\
& \quad+\oint_{S}\left\langle\boldsymbol{r} \cdot\left(\frac{\boldsymbol{E}_{g} \boldsymbol{E}_{g}+c^{2} \boldsymbol{B}_{g} \boldsymbol{B}_{g}}{4 \pi G}\right)+\boldsymbol{r} U_{g}\right\rangle \cdot d^{2} \boldsymbol{r} .
\end{aligned}
$$

Since at the edge of the fluid configuration the mass density $\rho$ and the pressure $p$ vanish (mass discontinuities are ignored) the virial theorem in equilibrium shows that the configuration is contained by the field surface terms. The field energy density existing beyond the fluid surface represents a back pressure acting on the fluid. Extending the integration volume to infinity, where the fields are assumed to vanish, it follows that

$$
\int_{V \rightarrow \infty}\left\langle\gamma \rho u^{2}+\left(3+\frac{\gamma_{A}}{\gamma_{A}-1} \gamma^{2} \beta^{2}\right) p+U_{g}\right\rangle d^{3} r=0 .
$$

Note that the gravitational energy density is negative, $U_{g}=-\left(E_{g}^{2}+c^{2} B_{g}^{2}\right) / 8 \pi G$, so that the fluid mass distribution is self-contained. In a non-equilibrium situation the terms involving the evolution of the average kinetic energy and of the moment of inertia must be taken into account, describing the expected collapse or expansion of clusters.

\section{GRAVITOELECTROMAGNETIC WAVES}

The Maxwell source equations give the gravitoelectromagnetic field in terms of the true mass density $\rho=m n$ and of the true mass current density $\boldsymbol{j}=\rho \boldsymbol{u}$ by

$$
\begin{aligned}
\boldsymbol{\nabla} \cdot \boldsymbol{E}_{g} & =-4 \pi G \rho & & \text { GEM Gauss's law } \\
\boldsymbol{\nabla} \times \boldsymbol{B}_{g} & =-\frac{4 \pi G}{c^{2}} \boldsymbol{j}+\frac{1}{c^{2}} \frac{\partial \boldsymbol{E}_{g}}{\partial t} & & \text { GEM Ampère's law }
\end{aligned}
$$

These equations satisfy the equation of continuity

$$
\frac{\partial \rho}{\partial t}+\nabla \cdot \boldsymbol{j}=0
$$

The gravitoelectromagnetic field can be also defined in terms of both the vector $\boldsymbol{A}_{g}$ and the scalar $\phi_{g}$ potentials:

$$
\begin{aligned}
\boldsymbol{B}_{g} & =\boldsymbol{\nabla} \times \boldsymbol{A}_{g}, \\
\boldsymbol{E}_{g} & =-\boldsymbol{\nabla} \phi_{g}-\frac{\partial \boldsymbol{A}_{g}}{\partial t},
\end{aligned}
$$

These definitions are a consequence of the homogeneous Maxwell equations

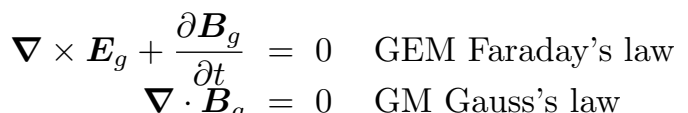

Accordingly, the source equations can be written in terms of the potentials as

$$
\begin{aligned}
\nabla^{2} \phi_{g}+\frac{\partial}{\partial t}\left(\boldsymbol{\nabla} \cdot \boldsymbol{A}_{g}\right) & =4 \pi G \rho \\
\left(\boldsymbol{\nabla}^{2} \boldsymbol{A}_{g}-\frac{1}{c^{2}} \frac{\partial^{2} \boldsymbol{A}_{g}}{\partial t^{2}}\right)-\boldsymbol{\nabla}\left(\boldsymbol{\nabla} \cdot \boldsymbol{A}_{g}+\frac{1}{c^{2}} \frac{\partial \phi_{g}}{\partial t}\right) & =\frac{4 \pi G}{c^{2}} \boldsymbol{j} .
\end{aligned}
$$


Since $\boldsymbol{B}_{g}$ is defined in terms of the rotational of $\boldsymbol{A}_{g}$, the gradient of a scalar function $f$ can be added to the vector potential without changing the value of $\boldsymbol{B}_{g}$ :

$$
\boldsymbol{A}_{g} \rightarrow \boldsymbol{A}_{g}+\nabla f
$$

The scalar potential must be simultaneously transformed to keep the GE field $\boldsymbol{E}_{g}$ unchanged

$$
\phi_{g} \rightarrow \phi_{g}+\frac{\partial f}{\partial t}
$$

This freedom means that the potentials can satisfy the Lorenz condition

$$
\boldsymbol{\nabla} \cdot \boldsymbol{A}_{g}+\frac{1}{c^{2}} \frac{\partial \phi_{g}}{\partial t}=0
$$

so that $\phi_{g}$ and $\boldsymbol{A}_{g}$ satisfy the wave equations

$$
\begin{aligned}
\nabla^{2} \phi_{g}-\frac{1}{c^{2}} \frac{\partial^{2} \phi_{g}}{\partial t^{2}} & =4 \pi G \rho \\
\boldsymbol{\nabla}^{2} \boldsymbol{A}_{g}-\frac{1}{c^{2}} \frac{\partial^{2} \boldsymbol{A}_{g}}{\partial t^{2}} & =\frac{4 \pi G}{c^{2}} \boldsymbol{j} .
\end{aligned}
$$

In general, the wave equation in the Lorenz gauge,

$$
\square^{2} \boldsymbol{A}_{g}=\frac{4 \pi G}{c^{2}} \boldsymbol{j}
$$

can be formally solved inverting the d'Alembertian operator

$$
\boldsymbol{A}_{g}(\boldsymbol{r}, t)=-\frac{G}{c^{2}} \int \frac{\boldsymbol{j}\left(\boldsymbol{r}^{\prime}, t^{\prime}\right)}{\left|\boldsymbol{r}-\boldsymbol{r}^{\prime}\right|} d^{3} r^{\prime} \quad \text { radiating regime }
$$

where $t^{\prime}=t-\left|\boldsymbol{r}-\boldsymbol{r}^{\prime}\right| / c$ is the retarded time. The solution depends not only on the distribution of elementary currents inside the source, but also on the relative velocities between different parts of the distribution. It is the difference in the retarded times which gives rise to radiation. Taking into account these phase differences, the fluid oscillations give rise to gravitoelectromagnetic waves, which propagate away to infinity. Such waves are also possible, for example, in the (Lorentz covariant) analogy put forward by Heaviside [5], and whose properties were described in a recent calculation of the gravitational radiation from orbiting binaries [35]. The waves associated with matter oscillations are not exclusive of the general relativity theory, but have different propagation characteristics that may depend on the particular stage of formation. The gravitoelectromagnetic waves here considered conceivably correspond to a weak phase of the gravitational waves.

Alternatively, the vector potential can satisfy the Coulomb gauge,

$$
\nabla \cdot \boldsymbol{A}_{g}=0
$$

so that the scalar potential satisfies the GE Poisson equation

$$
\nabla^{2} \phi_{g}=4 \pi G \rho
$$

According to this equation the scalar potential describes near field effects given in terms of the mass distribution by the instantaneous Coulomb's law solution

$$
\phi_{g}=-G \int \frac{\rho\left(\boldsymbol{r}^{\prime}, t\right)}{\left|\boldsymbol{r}-\boldsymbol{r}^{\prime}\right|} d^{3} r^{\prime} \text { for } \boldsymbol{\nabla} \cdot \boldsymbol{A}_{g}=0 .
$$

In the Coulomb gauge the vector potential satisfies the inhomogeneous wave equation

$$
\begin{aligned}
\boldsymbol{\nabla}^{2} \boldsymbol{A}_{g}-\frac{1}{c^{2}} \frac{\partial^{2} \boldsymbol{A}_{g}}{\partial t^{2}} & =\frac{4 \pi G}{c^{2}} \boldsymbol{j}+\frac{1}{c^{2}} \nabla \frac{\partial \phi_{g}}{\partial t} \\
& =\frac{G}{c^{2}}\left(4 \pi \boldsymbol{j}+\nabla \int \frac{\nabla^{\prime} \cdot \boldsymbol{j}\left(\boldsymbol{r}^{\prime}, t\right)}{\left|\boldsymbol{r}-\boldsymbol{r}^{\prime}\right|} d^{3} r^{\prime}\right)
\end{aligned}
$$


where the equation of mass continuity was used. The volume integral can be integrated by parts

$$
\int \frac{\boldsymbol{\nabla}^{\prime} \cdot \boldsymbol{j}\left(\boldsymbol{r}^{\prime}\right)}{\left|\boldsymbol{r}-\boldsymbol{r}^{\prime}\right|} d^{3} r^{\prime}=\int \boldsymbol{\nabla}^{\prime} \cdot\left(\frac{\boldsymbol{j}\left(\boldsymbol{r}^{\prime}\right)}{\left|\boldsymbol{r}-\boldsymbol{r}^{\prime}\right|}\right) d^{3} r^{\prime}-\int \boldsymbol{j}\left(\boldsymbol{r}^{\prime}\right) \cdot \nabla^{\prime}\left(\frac{1}{\left|\boldsymbol{r}-\boldsymbol{r}^{\prime}\right|}\right) d^{3} r^{\prime}
$$

Noting that

$$
\nabla^{\prime}\left(\frac{1}{\left|\boldsymbol{r}-\boldsymbol{r}^{\prime}\right|}\right)=-\nabla\left(\frac{1}{\left|\boldsymbol{r}-\boldsymbol{r}^{\prime}\right|}\right)
$$

the previous expression becomes

$$
\int \frac{\nabla^{\prime} \cdot \boldsymbol{j}\left(\boldsymbol{r}^{\prime}\right)}{\left|\boldsymbol{r}-\boldsymbol{r}^{\prime}\right|} d^{3} r^{\prime}=\oint \frac{\boldsymbol{j}\left(\boldsymbol{r}^{\prime}\right)}{\left|\boldsymbol{r}-\boldsymbol{r}^{\prime}\right|} \cdot d^{2} \boldsymbol{r}^{\prime}+\nabla \cdot \int \frac{\boldsymbol{j}\left(\boldsymbol{r}^{\prime}\right)}{\left|\boldsymbol{r}-\boldsymbol{r}^{\prime}\right|} d^{3} r^{\prime}
$$

Hence

$$
\begin{aligned}
\nabla^{2} \boldsymbol{A}_{g}-\frac{1}{c^{2}} \frac{\partial^{2} \boldsymbol{A}_{g}}{\partial t^{2}}= & \frac{G}{c^{2}}\left[4 \pi \boldsymbol{j}+\nabla \oint \frac{\boldsymbol{j}\left(\boldsymbol{r}^{\prime}, t\right)}{\left|\boldsymbol{r}-\boldsymbol{r}^{\prime}\right|} \cdot d^{2} \boldsymbol{r}^{\prime}+\nabla\left(\nabla \cdot \int \frac{\boldsymbol{j}\left(\boldsymbol{r}^{\prime}, t\right)}{\left|\boldsymbol{r}-\boldsymbol{r}^{\prime}\right|} d^{3} r^{\prime}\right)\right] \\
=\frac{G}{c^{2}}[ & 4 \pi \boldsymbol{j}+\nabla \oint \frac{\boldsymbol{j}\left(\boldsymbol{r}^{\prime}, t\right)}{\left|\boldsymbol{r}-\boldsymbol{r}^{\prime}\right|} \cdot d^{2} \boldsymbol{r}^{\prime} \\
& \left.+\boldsymbol{\nabla} \times\left(\boldsymbol{\nabla} \times \int \frac{\boldsymbol{j}\left(\boldsymbol{r}^{\prime}, t\right)}{\left|\boldsymbol{r}-\boldsymbol{r}^{\prime}\right|} d^{3} r^{\prime}\right)+\nabla^{2} \int \frac{\boldsymbol{j}\left(\boldsymbol{r}^{\prime}, t\right)}{\left|\boldsymbol{r}-\boldsymbol{r}^{\prime}\right|} d^{3} r^{\prime}\right] .
\end{aligned}
$$

The last term in the right-hand side can be simplified using the relation

$$
\nabla^{2}\left(\frac{1}{\left|\boldsymbol{r}-\boldsymbol{r}^{\prime}\right|}\right)=-4 \pi \delta^{3}\left(\boldsymbol{r}-\boldsymbol{r}^{\prime}\right)
$$

and used to cancel the first term. Therefore, in the Coulomb gauge $\boldsymbol{\nabla} \cdot \boldsymbol{A}_{g}=0$ the wave equation for $\boldsymbol{A}_{g}$ becomes $[32]$

$$
\nabla^{2} \boldsymbol{A}_{g}-\frac{1}{c^{2}} \frac{\partial^{2} \boldsymbol{A}_{g}}{\partial t^{2}}=\frac{G}{c^{2}}\left[\nabla \times\left(\nabla \times \int \frac{\boldsymbol{j}\left(\boldsymbol{r}^{\prime}, t\right)}{\left|\boldsymbol{r}-\boldsymbol{r}^{\prime}\right|} d^{3} r^{\prime}\right)+\nabla \oint \frac{\boldsymbol{j}\left(\boldsymbol{r}^{\prime}, t\right)}{\left|\boldsymbol{r}-\boldsymbol{r}^{\prime}\right|} \cdot d^{2} \boldsymbol{r}^{\prime}\right],
$$

where the first term on the right-hand side corresponds to the transverse mass current density distribution (source of internal gravitoelectromagnetic waves) and the second term to the contribution of surface currents (source of surface waves) which vanishes for limited sources. This equation describes the excitation and propagation of gravitoelectromagnetic waves. These can be either nonpropagating waves, that can be properly named gravity waves (fluid oscillations), or propagating gravitatoelectromagnetic waves. Note that

$$
\nabla^{2} \boldsymbol{A}_{g}=\boldsymbol{\nabla} \underbrace{\left(\boldsymbol{\nabla} \cdot \boldsymbol{A}_{g}\right)}_{0}-\nabla \times \nabla \times \boldsymbol{A}_{g}=-\nabla \times \nabla \times \boldsymbol{A}_{g}
$$

in the Coulomb gauge condition. Thus in the low-frequency regime the vector potential is given simply by the Biot-Savart's law

$$
\boldsymbol{A}_{g}(\boldsymbol{r}, t)=-\frac{G}{c^{2}} \int \frac{\boldsymbol{j}\left(\boldsymbol{r}^{\prime}, t\right)}{\left|\boldsymbol{r}-\boldsymbol{r}^{\prime}\right|} d^{3} r^{\prime} \quad \text { non-radiating regime }
$$

In the Coulomb gauge the scalar potential $\phi_{g}$ contributes only to the near fields, both in the low- and high-frequency regimes. The same is true for the vector potential $\boldsymbol{A}_{g}$ in the low-frequency case when only gravitoelectromagnetic waves may be formed which do not radiate. However, in the high-frequency regime the vector potential satisfies a wave equation with the transverse current density contributing to the formation of gravitoelectromagnetic waves over all space, even for localized sources.

\section{BOUNDARY CONDITIONS AND ENERGY DENSITY DISTRIBUTION}

The fluid and gravitoelectromagnetic field variables must satisfy several boundary conditions at the fluid-vacuum interface, as follows. Let $\boldsymbol{n}$ denote the unit vector normal to an interface and $\langle\langle X\rangle\rangle$ the increment of any quantity $X$ across the interface in the direction $\boldsymbol{n}$. For the interface between two fluids

$$
\boldsymbol{n} \cdot\langle\langle\boldsymbol{u}\rangle\rangle=0 \text {. }
$$


For the fluid-vacuum interface

$$
\boldsymbol{n} \cdot \boldsymbol{u}=0
$$

Gauss's law in the fluid-vacuum interface gives

$$
\begin{array}{ll}
\nabla \cdot \boldsymbol{E}_{g}=-4 \pi G \rho & \Longrightarrow \boldsymbol{n} \cdot\left\langle\left\langle\boldsymbol{E}_{g}\right\rangle\right\rangle=-4 \pi G \sigma \\
\nabla \cdot \boldsymbol{B}_{g}=0 & \Longrightarrow \boldsymbol{n} \cdot\left\langle\left\langle\boldsymbol{B}_{g}\right\rangle\right\rangle=0
\end{array}
$$

where $\sigma$ designates the surface mass density. Also, the laws of Faraday and Ampère give

$$
\begin{array}{ll}
\nabla \times \boldsymbol{E}_{g}=-\frac{\partial \boldsymbol{B}_{g}}{\partial t} & \Longrightarrow \boldsymbol{n} \times\left\langle\left\langle\boldsymbol{E}_{g}\right\rangle\right\rangle=0 \\
\nabla \times \boldsymbol{B}_{g}=-\frac{4 \pi G}{c^{2}} \rho \boldsymbol{u}+\frac{1}{c^{2}} \frac{\partial \boldsymbol{E}_{g}}{\partial t} \Longrightarrow \boldsymbol{n} \times\left\langle\left\langle\boldsymbol{B}_{g}\right\rangle\right\rangle=-\frac{4 \pi G}{c^{2}} \boldsymbol{K}
\end{array}
$$

where $\boldsymbol{K}$ is the surface mass current density.

Recall the equation of momentum density conservation derived in Subsection II A

$$
\rho \frac{d}{d t}\left[\gamma\left(1+\frac{\gamma_{A}}{\gamma_{A}-1} \frac{\gamma p}{\rho c^{2}}\right) \boldsymbol{u}\right]=-\nabla p+\rho\left(\boldsymbol{E}_{g}+\boldsymbol{u} \times \boldsymbol{B}_{g}\right) .
$$

Using the laws of Gauss, Ampère and Faraday this equation can be written as

$$
\begin{aligned}
& \rho \frac{d}{d t}\left[\gamma\left(1+\frac{\gamma_{A}}{\gamma_{A}-1} \frac{\gamma p}{\rho c^{2}}\right) \boldsymbol{u}\right] \\
& =-\nabla p+\frac{\partial}{\partial t}\left(\frac{\boldsymbol{E}_{g} \times \boldsymbol{B}_{g}}{4 \pi G}\right)-\frac{1}{4 \pi G}\left[\nabla \cdot\left(\boldsymbol{E}_{g} \boldsymbol{E}_{g}\right)-\left(\boldsymbol{E}_{g} \cdot \nabla\right) \boldsymbol{E}_{g}\right] \\
& \quad+\frac{1}{4 \pi G}\left[\boldsymbol{E}_{g} \times\left(\nabla \times \boldsymbol{E}_{g}\right)+c^{2} \boldsymbol{B}_{g} \times\left(\nabla \times \boldsymbol{B}_{g}\right)\right] .
\end{aligned}
$$

Using the following relations

$$
\left\{\begin{aligned}
\left(\nabla \cdot \boldsymbol{E}_{g}\right) \boldsymbol{E}_{g} & =\nabla \cdot\left(\boldsymbol{E}_{g} \boldsymbol{E}_{g}\right)-\left(\boldsymbol{E}_{g} \cdot \nabla\right) \boldsymbol{E}_{g}=\left(\nabla \boldsymbol{E}_{g}\right) \cdot \boldsymbol{E}_{g}-\left(\boldsymbol{E}_{g} \times \nabla\right) \times \boldsymbol{E}_{g} \\
\left(\nabla \cdot \boldsymbol{B}_{g}\right) \boldsymbol{B}_{g} & =\nabla \cdot\left(\boldsymbol{B}_{g} \boldsymbol{B}_{g}\right)-\left(\boldsymbol{B}_{g} \cdot \nabla\right) \boldsymbol{B}_{g}=0 \\
\boldsymbol{E}_{g} \times\left(\nabla \times \boldsymbol{E}_{g}\right) & =\left(\nabla \boldsymbol{E}_{g}\right) \cdot \boldsymbol{E}_{g}-\left(\boldsymbol{E}_{g} \cdot \nabla\right) \boldsymbol{E}_{g}=\frac{\nabla E_{g}^{2}}{2}-\left(\boldsymbol{E}_{g} \cdot \nabla\right) \boldsymbol{E}_{g} \\
\boldsymbol{B}_{g} \times\left(\nabla \times \boldsymbol{B}_{g}\right) & =\left(\nabla \boldsymbol{B}_{g}\right) \cdot \boldsymbol{B}_{g}-\left(\boldsymbol{B}_{g} \cdot \nabla\right) \boldsymbol{B}_{g}=\frac{\nabla B_{g}^{2}}{2}-\left(\boldsymbol{B}_{g} \cdot \nabla\right) \boldsymbol{B}_{g}
\end{aligned}\right.
$$

the equation of momentum density conservation becomes

$$
\begin{aligned}
\rho \frac{d}{d t}\left[\gamma\left(1+\frac{\gamma_{A}}{\gamma_{A}-1} \frac{\gamma p}{\rho c^{2}}\right) \boldsymbol{u}\right]= & \frac{\partial}{\partial t}\left(\frac{\boldsymbol{E}_{g} \times \boldsymbol{B}_{g}}{4 \pi G}\right) \\
& -\nabla \cdot\left[\left(p-\frac{E_{g}^{2}+c^{2} B_{g}^{2}}{8 \pi G}\right) \overline{\bar{I}}+\frac{\boldsymbol{E}_{g} \boldsymbol{E}_{g}+c^{2} \boldsymbol{B}_{g} \boldsymbol{B}_{g}}{4 \pi G}\right] .
\end{aligned}
$$

Furthermore, one may use the expansions

$$
\begin{aligned}
\rho \frac{d}{d t}\left[\gamma\left(1+\frac{\gamma_{A}}{\gamma_{A}-1} \frac{\gamma p}{\rho c^{2}}\right) \boldsymbol{u}\right]= & \rho \frac{\partial}{\partial t}\left[\gamma\left(1+\frac{\gamma_{A}}{\gamma_{A}-1} \frac{\gamma p}{\rho c^{2}}\right) \boldsymbol{u}\right] \\
& +\rho \gamma\left(1+\frac{\gamma_{A}}{\gamma_{A}-1} \frac{\gamma p}{\rho c^{2}}\right) \boldsymbol{u} \cdot \nabla \boldsymbol{u} \\
& +\rho u^{2} \nabla\left[\gamma\left(1+\frac{\gamma_{A}}{\gamma_{A}-1} \frac{\gamma p}{\rho c^{2}}\right)\right],
\end{aligned}
$$

and

$$
\begin{aligned}
\nabla \cdot\left(\frac{\boldsymbol{E}_{g} \boldsymbol{E}_{g}+c^{2} \boldsymbol{B}_{g} \boldsymbol{B}_{g}}{4 \pi G}\right) & =\frac{\left(\boldsymbol{E}_{g} \cdot \nabla\right) \boldsymbol{E}_{g}+c^{2}\left(\boldsymbol{B}_{g} \cdot \nabla\right) \boldsymbol{B}_{g}}{4 \pi G}-\rho \boldsymbol{E}_{g} \\
& =\frac{\left(\boldsymbol{E}_{g} \cdot \nabla\right) \boldsymbol{E}_{g}+c^{2}\left(\boldsymbol{B}_{g} \cdot \nabla\right) \boldsymbol{B}_{g}}{4 \pi G}+\rho\left(\nabla \phi_{g}+\frac{\partial \boldsymbol{A}_{g}}{\partial t}\right) .
\end{aligned}
$$


Denoting an infinitesimal displacement from one side of a fluid interface to the other by $\delta \boldsymbol{r}$, the equation of momentum density conservation gives

$$
\begin{array}{r}
\rho \frac{\partial}{\partial t}\left[\gamma\left(1+\frac{\gamma_{A}}{\gamma_{A}-1} \frac{\gamma p}{\rho c^{2}}\right) \boldsymbol{u} \cdot \delta \boldsymbol{r}\right]+\rho \gamma\left(1+\frac{\gamma_{A}}{\gamma_{A}-1} \frac{\gamma p}{\rho c^{2}}\right)(\boldsymbol{u} \cdot \nabla \boldsymbol{u}) \cdot \delta \boldsymbol{r} \\
+\rho u^{2} \delta\left[\gamma\left(1+\frac{\gamma_{A}}{\gamma_{A}-1} \frac{\gamma p}{\rho c^{2}}\right)\right]=\delta \boldsymbol{r} \cdot \frac{\partial}{\partial t}\left(\frac{\boldsymbol{E}_{g} \times \boldsymbol{B}_{g}}{4 \pi G}\right)-\delta\left(p-\frac{E_{g}^{2}+c^{2} B_{g}^{2}}{8 \pi G}\right) \\
-\left(\frac{\left(\boldsymbol{E}_{g} \cdot \nabla\right) \boldsymbol{E}_{g}+c^{2}\left(\boldsymbol{B}_{g} \cdot \nabla\right) \boldsymbol{B}_{g}}{4 \pi G}\right) \cdot \delta \boldsymbol{r}-\rho \delta \phi_{g}-\rho \delta \boldsymbol{r} \cdot \frac{\partial \boldsymbol{A}_{g}}{\partial t} .
\end{array}
$$

Taking the limit $\delta \boldsymbol{r} \rightarrow 0$

$$
\rho u^{2} \delta\left[\gamma\left(1+\frac{\gamma_{A}}{\gamma_{A}-1} \frac{\gamma p}{\rho c^{2}}\right)\right]+\delta\left(p-\frac{E_{g}^{2}+c^{2} B_{g}^{2}}{8 \pi G}\right)+\rho \delta \phi_{g}=0
$$

Hence

$$
\left\langle\left\langle\rho u^{2}\left[\gamma\left(1+\frac{\gamma_{A}}{\gamma_{A}-1} \frac{\gamma p}{\rho c^{2}}\right)\right]+p+\rho \phi_{g}+U_{g}\right\rangle\right\rangle=0 .
$$

This indicates that the sum of the kinetic energy density and the pressure are balanced across each interface both by the negative GE potential $\phi_{g}$ and by the negative gravitoelectromagnetic field energy density $U_{g}$.

\section{COMMENTS AND CONCLUSIONS}

A consistent set of hydrodynamic and Maxwell equations for the gravitoelectromagnetic field was obtained applying Hamilton's principle to a perfect fully-relativistic fluid in flat space, leading to an extended gravitoelectromagnetic or hydrogravitoelectromagnetic (EGEM or HGEM) model. The variational principle was carried out both in the Eulerian and Lagrangian frameworks. The Eulerian formulation in the mean fluid variables leads to equations which describe the conservation of energy and momentum for fluid flow immersed in a self-consistent or external gravitoelectromagnetic field. The Lagrangian formulation, in particular, gives an equation for the rate of change of the canonical momentum of a fluid element (particle). Furthermore, the momentum densities canonically conjugate to the Lagrangian field coordinates allow to define the Hamiltonian density and an energy integral for the fully relativistic flow in the gravitoelectromagnetic field.

The derived equations of motion for a relativistic fluid constitute an extended version of the gravitomagnetic theory originally proposed by Thirring [12] for a test mass within the weak general relativistic formulation, although using a different approach. The present extended version is adequate for the analysis, in flat space, of phenomena such as the galactic rotation curve [18]. However, it does not describe effects which depend on higher order terms in the velocity of light, in the Lorentz force. These terms are needed, for example, to calculate the relativistic correction to the planetary perihelion shift. Nevertheless, it removes many restrictions of the original theory, which was limited to quasi-static solutions and did not include the fluid pressure and the full relativistic inertial and thermal corrections. The long-standing question about the validity of Faraday's law in the gravitomagnetic theory is also naturally solved.

It was demonstrated that the equations for fluid motion contain a Cauchy invariant, which partially integrates the equations of motion and has important consequences in astrophysical applications, as it includes the vorticity inducing and braking effects of the gravitomagnetic field. These effects are illustrated in the study of the rotational motion of galaxies [18]. The braking effect is also manifested in the anomalous precession of the planetary perihelion (cf. the joint paper "Extended gravitoelectromagnetism. III. Mercury's perihelion precession"). The conservation equations of fluid flow were written in compact form by the definition of the momentum, stress and energy densities for both the fluid and the field. This allows to write the total momentum and energy conservation equations in covariant form. The integral forms of the conservation equations were also derived with emphasis in a general form of the virial theorem. The equations which describe the excitation and propagation of gravitoelectromagnetic waves according to the standard gauge conditions were discussed, as well as the boundary conditions and the energy density distribution in the fluid.

Note that the electromagnetic field theory gives a result similar to the gravitoelectromagnetic field equations with the following analogies

$$
\begin{aligned}
\boldsymbol{E} & \rightarrow \boldsymbol{E}_{g} \quad, \boldsymbol{B} \rightarrow \boldsymbol{B}_{g} \\
\phi & \rightarrow \phi_{g} \quad, \boldsymbol{A} \rightarrow \boldsymbol{A}_{g} \\
\epsilon_{0} & \rightarrow-\frac{1}{4 \pi G}, \mu_{0} \rightarrow-\frac{4 \pi G}{c^{2}}
\end{aligned}
$$


and the charge $q$ replaced by the mass $m$. In this way, the results of the present paper are entirely similar to the results presented in [19]. However, there are many important differences, since the gravitoelectromagnetic field is only attractive. This is manifested, for example, in the energy integral and in the virial theorem.

Last, the covariant equations which describe the interaction between the fluid and the gravitoelectromagnetic field in flat space make it possible to obtain the perturbations in the metric tensor according to the weak general relativity formulation, but this is the subject of the second part article in the present three-parts work (cf. the joint paper "Extended gravitoelectromagnetism. II. Metric perturbation").

\section{ACKNOWLEDGMENTS}

The author acknowledges useful discussions with Rubens de Melo Marinho Jr. and Manuel Máximo Bastos Malheiro de Oliveira. This work was supported by a grant provided by the Programa de Capacitação Institucional: Diretoria de Pesquisa e Desenvolvimento/Comissão Nacional de Energia Nuclear (CNEN).

[1] M.F. Tisserand. Les travaux de Le Verrier. Annales de l'Observatoire de Paris, Mémoires, 15:23-43, 1880.

[2] J.C. Maxwell. Note on the attraction of gravitation, in: A dynamical theory of the electromagnetic field. Phil. Trans. R. Soc. Lond., 155:459-512, 1865. Page 492-493.

[3] G. Holzmüller. Ueber die Anwendung der Jacobi-Hamilton'schen Methode auf den Fall der Anziehung nach dem electrodynamischen Gesetze von Weber. Zeitschr. Math. Phys., XV:69-91, 1870.

[4] M.F. Tisserand. Sur le mouvement des planètes autour du Soleil, d'après la loi électrodynamique de Weber. Comptes Rendus Acad. Sci., 75:760-763, 1872.

[5] O. Heaviside. A gravitational and electromagnetic analogy, in: Electromagnetic Theory Vol.I. The Electrician, London, 1893. Pages 455-466.

[6] A. Einstein. Die Grundlage der allgemeine Relativitätstheorie. Ann. Phys., 354:769-822, 1916.

[7] A. Einstein. The meaning of relativity. Princeton University Press, Princeton, NJ, fifth edition, 1956.

[8] W. Pauli. Theory of relativity. Pergamon Press, London, 1958.

[9] H. Thirring. Über die Wirkung Rotierender Ferner Massen in der Einsteinschen Gravitationstheorie. Phys. Zeit., 19:33-39, 1918.

[10] J. Lense and H. Thirring. Über den Einflu $\beta$ der Eigenrotation der Zentralkörper auf die Bewegung der Planeten und Monden nach der Einsteinschen Gravitationstheorie. Phys. Zeit., 19:156-163, 1918.

[11] H. Pfister. On the history of the so called Lense-Thirring effect. Gen. Relat. Gravit., 39:1735-1748, 2007.

[12] H. Thirring. Über die formale Analogie zwischen den elektromagnetischen Grundgleichugen und den Einsteischen Gravitationsgleichungen erster Näherung. Phys. Zeit., 19:204-205, 1918.

[13] H. Pfister. Editorial note to: Hans Thirring, on the formal analogy between the basic electromagnetic equations and Einstein's gravity equations in first approximation. Gen. Relat. Gravit., 44:3217-3224, 2012.

[14] B. Mashoon. Gravitoelectromagnetism: a brief review, arxiv:031103v2 [gr-qc]. 2008.

[15] H.C. Ohanian and R. Ruffini. Gravitation and Spacetime. Cambridge University Press, Cambridge, third edition, 2013.

[16] T.A. Moore. A general relativity workbook. University Science Books, Mill Valley, CA, 2013.

[17] A. Bakopoulos and P. Kanti. Novel ansatzes and scalar quantities in gravitoelectromagnetism. Gen. Relativ. Gravit., 49:44-73, 2017

[18] G.O. Ludwig. Galactic rotation curve without dark matter. 2020. Submitted for publication.

[19] G.O. Ludwig. Variational formulation of plasma dynamics. Phys. Plasmas, 27:022110(21), 2020.

[20] J.W. Herivel. The derivation of the equations of motion of an ideal fluid by Hamilton's principle. Proc. Cambridge Phil. Soc., 51:344-349, 1955.

[21] J. Serrin. Mathematical principles of classical fluid mechanics. Handbuch der Physik, Vol. 3/8/1:125-263, 1959.

[22] R.L Seliger and G.B. Witham. Variational principles in continuum mechanics. Proc. R. Soc. Lond. A, 305:1-25, 1968.

[23] R. Salmon. Hamiltonian fluid mechanics. Ann. Rev. Fluid Mech., 20:225-256, 1988.

[24] G. Badin and F. Crisciani. Variational Formulation of Fluid and Geophysical Fluid Dynamics - Mechanics, Symmetries and Conservation Laws. Springer International Publishing AG, Cham, Switzerland, 2018.

[25] G. Webb. Magnetohydrodynamics and Fluid Dynamics: Action Principles and Conservation Laws. Springer International Publishing AG, Cham, Switzerland, 2018.

[26] H. Goldstein, C.P. Poole, and J.L. Safko. Classical Mechanics. Addison Wesley, Boston, MA, third edition, 2001.

[27] L. Comisso and F.A. Asenjo. Thermal-inertial effects on magnetic reconnection in relativistic pair plasmas. Phys. Rev. Lett., 113:045001(5), 2014.

[28] A.L. Cauchy. Théorie de la propagation des ondes à la surface d'un fluide pesant d'une profondeur indéfinie. Mém. Divers Savants, 1:5-318, 1815.

[29] U. Frisch and B. Villone. Cauchy's almost forgotten Lagrangian formulation of the Euler equation for 3D incompressible flow. arXiv:1402.4957v3 [math.HO], 2014. 
[30] S. Weinberg. Gravitation and cosmology: principles and applications of the general theory of relativity. John Wiley and Sons, New York, NY, 1972.

[31] W.K.H. Panofsky and M. Phillips. Classical electricity and magnetism. Addison-Wesley, Reading, MA, second edition, 1962.

[32] J.D. Jackson. Classical electrodynamics. John Wiley and Sons, Hoboken, NJ, third edition, 1998.

[33] J.A. Stratton. Electromagnetic theory. McGraw-Hill, New York, NY, 1941.

[34] L.D. Landau and E.M. Lifshitz. The classical theory of fields. Butterworth-Heinemann - Reed Elsevier, Oxford, fourth revised english edition, 1996.

[35] R.C. Hilborn. Gravitational waves from orbiting binaries without general relativity. Am. J. Phys., 86:186-197, 2018. 